Chinese Journal of Organic Chemistry

\title{
三亚吡嗪材料能源应用研究进展
}

\author{
崔超慧刘玉婷＼cjkstart杜 亚* \\ (南京工业大学化学与分子工程学院 先进化学制造研究院 南京 211816)
}

\begin{abstract}
摘要 三亚吡嗪(HAT)是一种缺电子刚性平面芳香族盘状结构, 具有出色的 $\pi-\pi$ 堆叠能力. 由于独特的拓扑结构和电子 特征, HAT 已被广泛用于构筑超分子材料、共价有机框架材料(COFs)、多孔氢键有机框架材料(HOFs)、金属有机框架 材料(MOFs)等. HAT 衍生物在催化、半导体、单分子磁体、水氧化、质子传导等方面也表现出巨大的应用潜能. 近年 来, 由于能源需求的激增, 科学家们基于 HAT 衍生物在能源领域中的应用进行了大量的研究. 在跟踪了 HAT 衍生物在 能源领域的研究进展的基础上，综述了该领域研究的最新进展.
\end{abstract}

关键词 三亚吡嗪(HAT)衍生物; 能源应用; 共价有机框架

\section{Recent Advancements of Hexaazatriphenylene-Based Materials for Energy Applications}

\author{
Cui, Chaohui $\quad$ Liu, Yuting $\quad$ Du, Ya* \\ (Institute of Advanced Synthesis, School of Chemistry and Molecular Engineering, Nanjing Tech University, Nanjing 211816)
}

\begin{abstract}
Hexaazatriphenylene (HAT) is an electron deficient, rigid, planar, aromatic discotic molecule with three fused pyrazine rings and excellent $\pi-\pi$ stacking ability. Due to its excellent topology and electronic properties, HAT has been exploited as structural motifs of supramolecules, covalent organic frameworks (COFs), porous hydrogen-bonded organic frameworks (HOFs), and metal organic frameworks (MOFs). HAT derivatives have been utilized in catalysis, semiconductors, monomolecular magnets, water oxidation, proton conduction, etc. In recent years, motivated by the increasing energy demand, scientists have intensively studied the energy applications of HAT derivatives. In this paper, the recent progress of HAT derivatives in the field of energy has been reviewed.
\end{abstract}

Keywords tripyrazine (HAT) derivatives; energy application; covalent organic framework

三亚吡嗪(Hexaazatriphenylene, HAT) (Scheme 1), 由三个稠合的吡嗪环构成, 具有良好的对称性, $\mathrm{sp}^{2}$ 杂化 作用使它拥有出色的电子缺陷性质和高 $\pi-\pi$ 堆积趋势. 作为刚性且平面的氮杂芳香族骨架, HAT 是最小二维含 氮的多环芳香族体系. 1981 年 Nasielski-Hinkens 等 ${ }^{[1]}$ 报 道了 HAT 的合成, 由于结合了独特拓扑结构和电子特 征以及强配位能力, HAT 吸引了科学家们的研究兴趣, 不断涌现出基于 HAT 的聚合物、大分子、配合物等. 近 年来, 随着有机多孔材料 ${ }^{[2]}$ 的发展, HAT 作为构建新型 多孔聚合物的明星构建基块 ${ }^{[3]}$ 被广泛用于构筑新型金属 配合物 ${ }^{[4]}$ 、固有微孔聚合物(PIMs) ${ }^{[5]}$ 、共轭微孔聚合物<smiles>c1cnc2c(n1)c1nccnc1c1nccnc21</smiles>

图式 1 HAT 的结构

Scheme 1 Structure of HAT

$(\mathrm{CMPs})^{[6]}$ 、多孔氢键有机框架 $(\mathrm{HOFs})^{[7]}$ 和共价有机框架 $(\mathrm{COFs})^{[8]}$. 通过对 HAT 的修饰和构建, HAT 衍生物已被 广泛应用于质子传导 ${ }^{[9]}$ 、催化剂[10]、半导体 ${ }^{[1]]}$ 、单分子

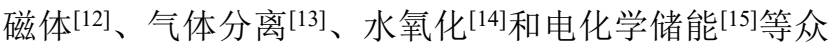

\footnotetext{
* Corresponding author. E-mail: ias_ydu@njtech.edu.cn

Received May 17, 2021; revised July 31, 2021; published online August 17, 2021.

Project supported by the National Natural Science Foundation of China (No. 21805134), the Natural Science Foundation of Jiangsu Province (No. BK20191363), the Science and Technology Innovation Project for Overseas Students from Nanjing City, and the Start-up Grant from Nanjing Tech University (No. 39837141).

国家自然科学基金(No. 21805134)、江苏省自然科学基金(No. BK20191363)、南京市留学人员择优资助、南京工业大学启动基金(No. 39837141)资助 项目.
} 
多领域. 目前, 关于 HAT 衍生物的综述主要关注其结构 设计与合成以及其在器件、多孔有机材料和金属配合物 等方面的应用，尚未涉及在能源应用领域的总结. 为此, 本综述对 HAT 衍生物在能源领域(如锂、钠、镁等离子 电池、钙钛矿电池和电容器)进行了总结, 重点介绍了 $\mathrm{HAT}$ 衍生物在电化学储能领域的研究进展.

化石资源的短缺、电动汽车 $(\mathrm{EV})$ 的快速增长以及可 再生能源的电网整合对能源储存和使用提出了迫切需 求 ${ }^{[16]}$. 电化学储能(Electrochemical Energy Storage, EES) 是一种基于电化学储能原理的技术, 具有无污染、用途 广泛、寿命长和维护成本低等优点, 推动了从便携式电 子产品到重型电动汽车, 甚至间歇性输配电电网等领域 的显著发展 ${ }^{[17]}$. 运用电化学储能技术提高储能设备的 性能和环境友好性将会是研究人员坚持不解的目标.

\section{HAT 衍生物在离子电池方面的应用}

有机材料作为电池和电容器电极具有环保、廉价、 安全、容易合成和可调控性强的优点 ${ }^{[18]}$. 传统上用于锂 离子电池电极的材料是基于过渡金属的无机材料, 例如 钴、铁、锡或镇等, 这些日益稀缺的矿产资源的大量使 用不满足可持续发展的要求, 并且无机配合物在提取和 合成过程中会放出有毒物质和大量金属废弃物, 需要消 耗大量能量. 因而有机材料作为锂离子电池电极材料的 研究引起了科学家们的广泛兴趣 ${ }^{[19]}$. 基于 HAT 单元的 构筑和修饰理论上会得到优良性质的金属离子电池电 极材料. 这主要是因为: (1) HAT 中存在多个缺电子结构 的吡嗪位点可供金属离子嵌入/脱嵌, 从而提供了高理 论比容量; (2) HAT 结构的 $\pi-\pi$ 共轭平面有利于电子和离 子的快速传输, 从而增强有机电极材料的导电性; (3) HAT 的平面盘状对称结构有利于构建具有二维 $\pi-\pi$ 共轭 扩展平面的聚合物, 以避免 HAT 单体在电解液中的溶 解, 得到具有长期稳定循环性能的电极材料. 早在 2003 年, Ohsaka 等 ${ }^{[20]}$ 率先通过循环伏安法对 HAT 的电化学 行为进行了研究, 他们给出了 HAT 内核的几种还原过 程 $\left(E^{1}{ }_{\mathrm{RED}}=-1.44 \mathrm{~V}, E^{2}{ }_{\mathrm{RED}}=-1.64 \mathrm{~V}\right.$ 和 $E_{\mathrm{RED}}^{3}=$ $-2.10 \mathrm{~V})$, 这归因于 HAT 具有多个电子不足的吡嗪位 点，可连续发生多个氧化还原反应.

\section{$1.1 \mathrm{HAT}$ 衍生物作为锂离子电池电极材料}

锂离子(Li-ion)电池自从索尼 20 世纪 90 年代初推出 以来, 就一直在电池市场上处于领先地位. 锂离子电池 (LIB)具有高倍率、高比能、高比功率、长循环寿命和快 速响应等优点, 从综合性能角度看, LIB 是目前最好的 电化学储能技术 ${ }^{[21]} .2011$ 年, Takayuki 课题组 ${ }^{[22]}$ 将 HAT 衍生物 $1,6,7,12,13,18$-六氮杂三荎撑(HATN)作为活性材 料, 以 $10 \%$ 的 HATN 与 $80 \%$ 的导电剂混合作为锂离子电
池正极材料, 以 $\mathrm{LiPF}_{6}-\mathrm{EC} / \mathrm{DEC}$ 为电解液进行电化学测 试, 结果显示 HATN 具有清晰分明的两对氧化还原峰 (图 1a), 对应于两步氧化还原反应, 作者推测第一步和 第二步的氧化还原反应可能分别涉及两个和四个电子 的转移, 测得电池第一圈的实际放电容量高达 420 $\mathrm{mAh} / \mathrm{g}$, 但 HATN 在电解液中的溶解导致第二圈和第三 圈的容量大幅度下降(图 $1 b$ ).
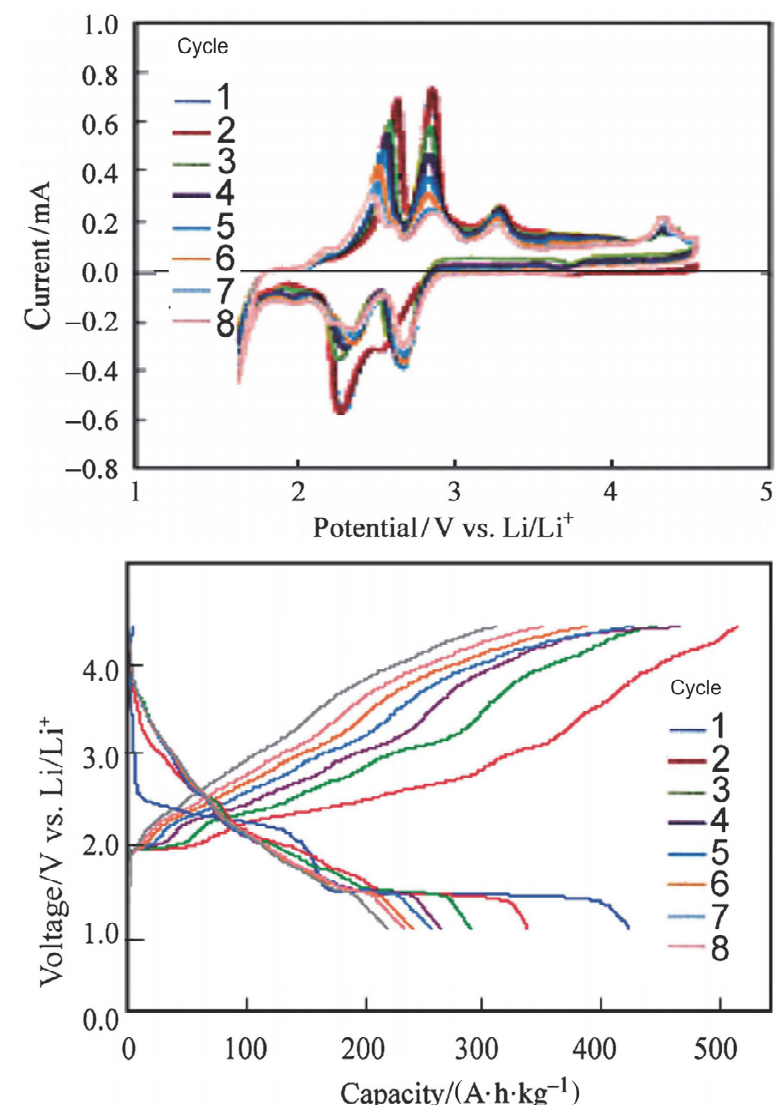

图 1 HATN 的电化学性能测试 [22]

Figure 1 HATN electrochemical performance test (a) Electrode CVs; (b) charge-discharge cycles for HATN

为了更深入探究 HATN 分子在充放电时的电化学 机理, 2017 年, Loh 课题组 ${ }^{[23]}$ 合成了具有 ${ }^{15} \mathrm{~N}$ 同位素标记 的 HATN(文章中命名为 $3 \mathrm{Q}$ ), 利用 ${ }^{15} \mathrm{~N}$ 相对较高的自然 丰度 $(0.37 \%)$ 和较小的自旋矩 $(I=1 / 2)$, 通过交叉极化/魔 角旋转 $(\mathrm{CP} / \mathrm{MAS})$ 固体核磁分析了锂化/去锂化过程中环 境的变化, 结合固态核磁氮谱、固态核磁碳谱和密度泛 函理论(DFT), 阐明了锂化过程中 HATN (3Q)的结构顺 序演变, 探测了芳族吡嗪氧化还原中心的锂化动力学, 从而证实在 HATN (3Q)中支持多电子转移(图 $2 a, 2 b$ ). 与 Takayuki 课题组推测结果不同, Loh 等 ${ }^{[23]}$ 计算出 HATN (3Q)具有两个氧化还原电势水平, 表现为两个三 电子过程, 根据两步三电子转移机理, 计算出的电位显 示为 $2.6 \sim 2.15 \mathrm{~V}$ (标记为“1-2-3”) 和 $1.68 \sim 1.38 \mathrm{~V}$ (标记为 
(a)

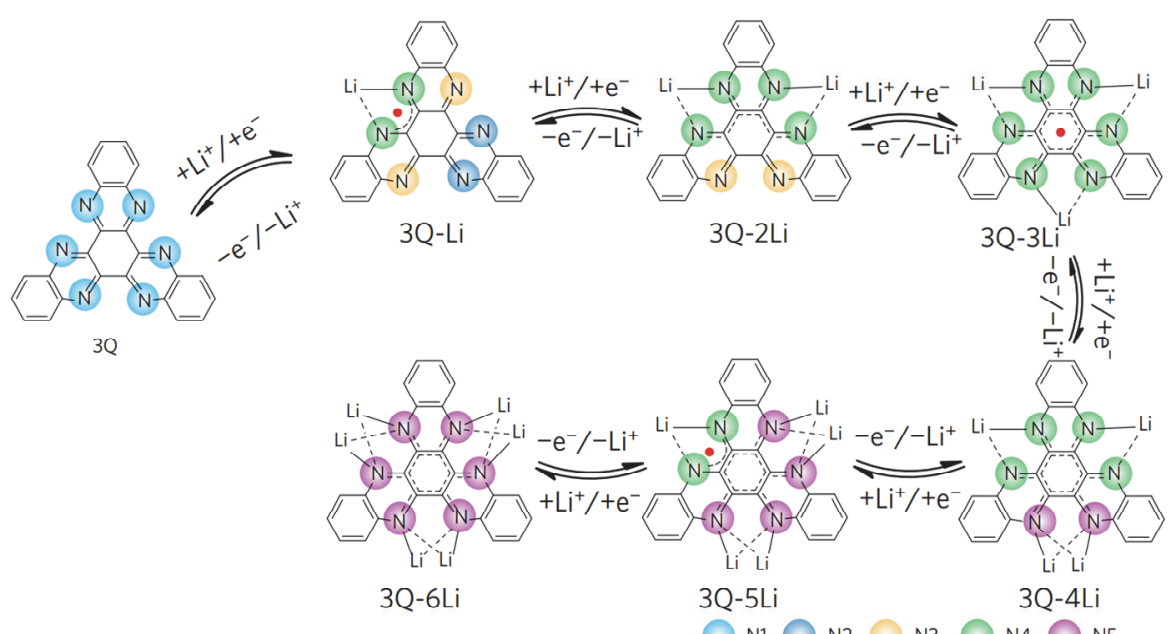

(b)

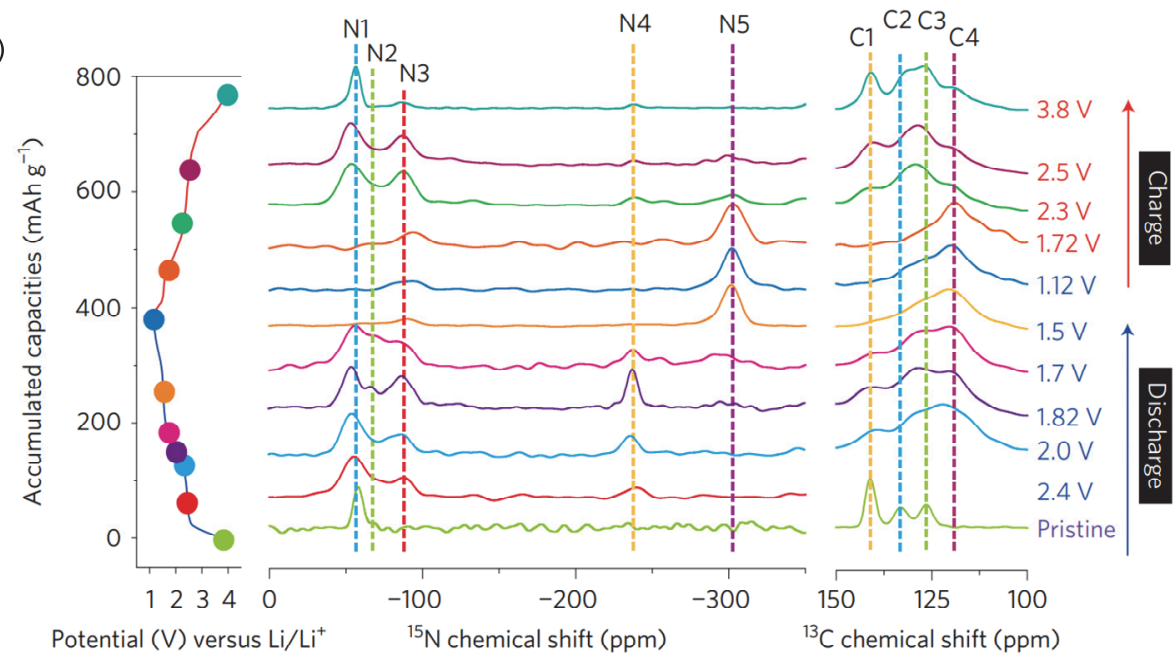

(c)

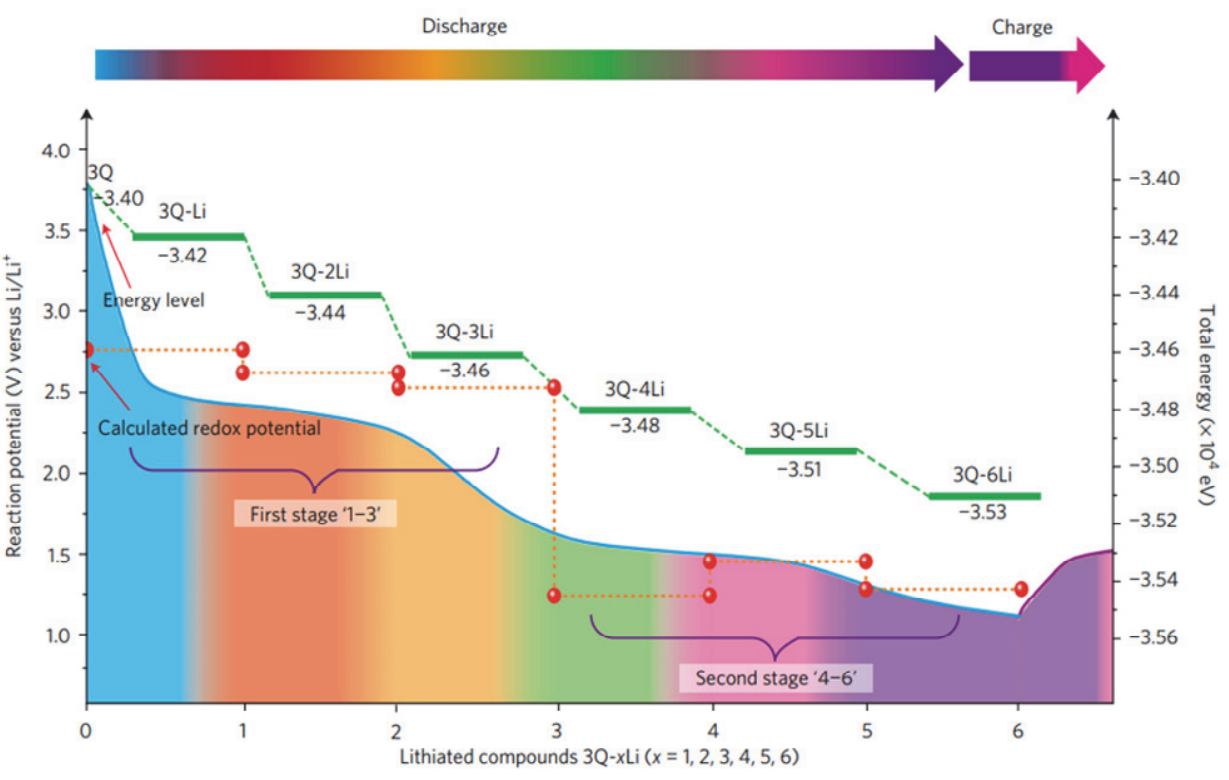

图 $23 \mathrm{Q}$ 充放电机理探究 ${ }^{[23]}$

Figure 2 Research on the charging and discharging mechanism of $3 \mathrm{Q}$

(a) The predicted lithiated structures of $3 \mathrm{Q}$ during the discharging process. (b) The galvanostatic charge/discharge profile of $3 \mathrm{Q} / \mathrm{Li}$ cycled at a rate of 20 $\mathrm{mA} / \mathrm{g}$ during the second cycle and ex-situ ${ }^{15} \mathrm{~N}$ and ${ }^{13} \mathrm{C}$ solid-state NMR spectra, respectively, of the electrode materials cycled to different charge states, as marked on the corresponding electrochemical profiles. (c) The lithiation pathway obtained from simulations 
“4-5-6”)的两个主放电平台(图 2c), 并制备了基于 HATN (3Q) $(30 \%)$ /石墨烯 $(60 \%)$ 混合正极材料, 在醚类电解液 [LiTFSI-DOL (1,3-二氧戊环)/DME (1,2-二甲氧基乙烷)] 中表现出良好的电化学性能: 在电流密度为 $0.4 \mathrm{~A} / \mathrm{g}$ 、 $1.2 \sim 3.9 \mathrm{~V}$ 的电压范围内显示为高达 $395 \mathrm{mAh} / \mathrm{g}$ 的放电 容量, 并在超高电流密度 $8 \mathrm{~A} / \mathrm{g}$ 时显示 $218 \mathrm{mAh} / \mathrm{g}$ 的比 容量(相当于理论比容量的 $52 \%$ ), 10000 次循环后保持 $67 \%$ 的容量, 优秀的放电容量、高倍率性能和极佳的长 循环稳定性使 HATN 电极在小分子锂离子电池电极材 料中表现地相当出色.

小分子 HATN 在电解液中的溶解会导致循环性能 变差, 且 HATN 分子作为电极材料时通常需要结合大量 的导电剂, 相当于增加了电池组分中惰性物质的重量, 这些对于电池比能量的提高是不利的. 为了降低小分子 在电解液中的溶解并减少导电剂的使用量, 2018 年张玉 根课题组 ${ }^{[24]}$ 将活性物质(50\%)和氧化石墨烯(GO, 40\%) 的混合物置于乙醇中超声, 社除溶剂后得到了锂离子电 池的复合正极材料 HATN/GO 和 HATNTA(六氮杂三荎 撑三羊酸 $/ \mathrm{GO}$. HAT 衍生物与 $\mathrm{GO}$ 表现出强烈的 $\pi-\pi$ 堆 积和氢键相互作用, 显著抑制了它们在电解液中的溶 解, 提高了电池正极的电导率和稳定性, 大大改善了电 化学性能. 该复合材料在使用市售的酯类 $\mathrm{LiPF}_{6}$ $\mathrm{EC}($ 碳酸乙烯酯 $) / \mathrm{DEC}$ (碳酸二乙酯) 电解液时具有出色 的长期循环稳定性, 在 $0.5 \mathrm{~A} / \mathrm{g}$ 的电流密度下 2000 次循 环后, HATN/GO 复合材料放电容量为 $122 \mathrm{mAh} / \mathrm{g}$, 保留 了 $80 \%$ 的初始容量; HATNTA/GO 复合材料放电容量为 $110 \mathrm{mAh} / \mathrm{g}$, 保留了 $86 \%$ 初始容量.

除了利用制备复合材料来降低小分子在电解液中 的溶解度来提高电极材料的循环稳定性之外, 解决溶解 问题的另一种常用策略是构筑聚合物材料. 2014 年, 江 东林课题组 ${ }^{[25]}$ 通过 Sonogashira 交叉偶联缩聚 1,4-二乙 炔基苯和 HATN-6I 制备了具有内置氧化还原活性单元 和永久纳米孔的共轭微孔聚合物 HATN-CMP(图 3a). 共 轭微孔聚合物的结构特征与锂离子电池在储能方面是 协同工作的, HATN-CMP 包含 HAT 单元, 这些氧化还原 活性单元充当了储能和供能模块; CMP 具有固有的开放 纳米孔, 可以使这些氧化还原位点充分接触到锂离子, 并且高的表面积有利于电荷动力学. 研究比较了 HATN-CMP 和 HATN 分别作为活性物质, 在相同的电 化学条件下[电压测试范围为 $1.5 \sim 4.0 \mathrm{~V}$; HATN-CMP/ HATN, 乙炔黑, PVDF 比例为 $6: 3: 2$; 电解液为 $\mathrm{LiPF}_{6}-\mathrm{EC} / \mathrm{DMC}$ (碳酸二甲酯)], HATN-CMP 的实际可逆 容量达 $147 \mathrm{mAh} / \mathrm{g}$, 为理论比容量的 71\%; HATN 初始放 电容量为 $52 \mathrm{mAh} / \mathrm{g}$, 仅为理论容量的 $56 \%$. 如图 $3 \mathrm{~b} 、 3 \mathrm{c}$ 所示, HATN-CMP 作为锂离子电池正极材料具有比
HATN 更高的实际容量和稳定性.

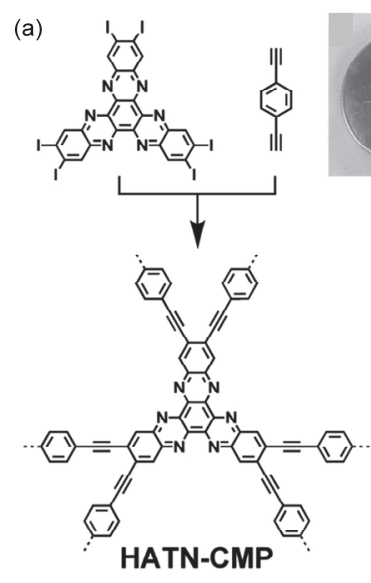

(b)

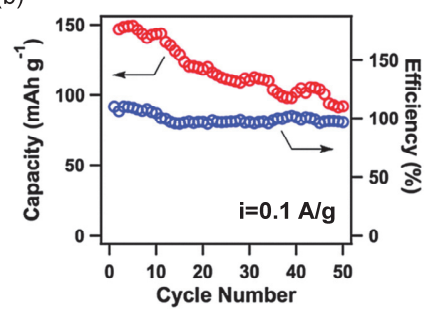

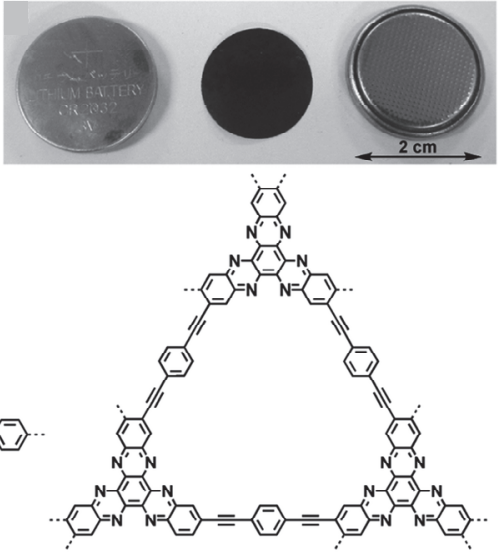

(c)
图 3 HATN-CMP 的结构及电化学性能表征 ${ }^{[25]}$

Figure 3 Characterization of structure and electrochemical performance of HATN-CMP

(a) Schematic representation of the synthesis of hexaazatrinaphthalene CMP (HATN-CMP), the elementary pore structure and the photos of HATN-CMP electrodes and lithium batteries thus fabricated. (b) Cycle stability of HATN-CMP. (c) Capacity retention ratio of HATN-CMP (filled circle) and monomer (open circle) cathode electrodes

将具有氧化还原活性位点的有机小分子引入合适 的多孔有机框架中，可以提高离子扩散速度和电子传导 性，同时降低其在电解液中的溶解度，但构建用于锂电 电极材料的多孔有机框架聚合物应综合考虑多种因素, 例如理论比容量、表面积、共轭度、孔径大小等. 尽管 HATN-CMP 具有高孔隙率, 但 HATN-CMP 表现出中等 的循环稳定性 (50 次循环后容量保持率为 $62 \%$ ) 和速率性 能 $(500$ 与 $100 \mathrm{~mA} / \mathrm{g}$ 电流密度下相比, 容量保持率仅为 44\%). 为了进一步探索 HAT 衍生物材料在锂离子电池 中的潜力, 2018 年张玉根课题组 ${ }^{[26]}$ 在氩气氛围下以环己 六酮水合物和 3,3'-二氨基联苯胺作为单体，合成了刚性 共轭框架材料 HATNPF1; 以环己六酮水合物和 3,3',4,4'四氨基二苯基甲烷为单体，合成了柔性非共轭框架材料 HATNPF2(图 4a), HATNPF1 的刚性框架使聚合物结构 在充放电过程中牢固而稳定. 在电化学测试中(电压测 试范围为 $1.5 \sim 4.0 \mathrm{~V}$; HATNPF1/HATNPF2, GO, PVDF 的比例为 $4: 5: 1$; 电解液为 $\mathrm{LiCF}_{3} \mathrm{SO}_{3} / \mathrm{G} 4$ 四甘醇二甲 醚盐), HATNPF 1 表现出高达 $309 \mathrm{mAh} / \mathrm{g}$ 的放电 
a

b

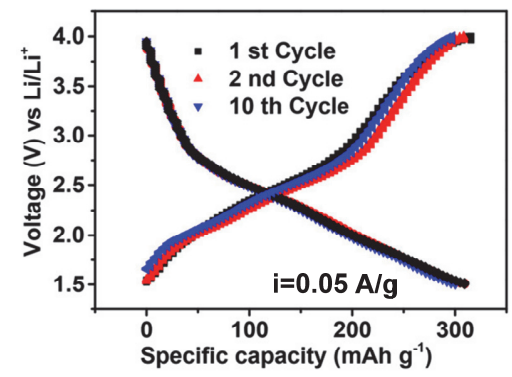

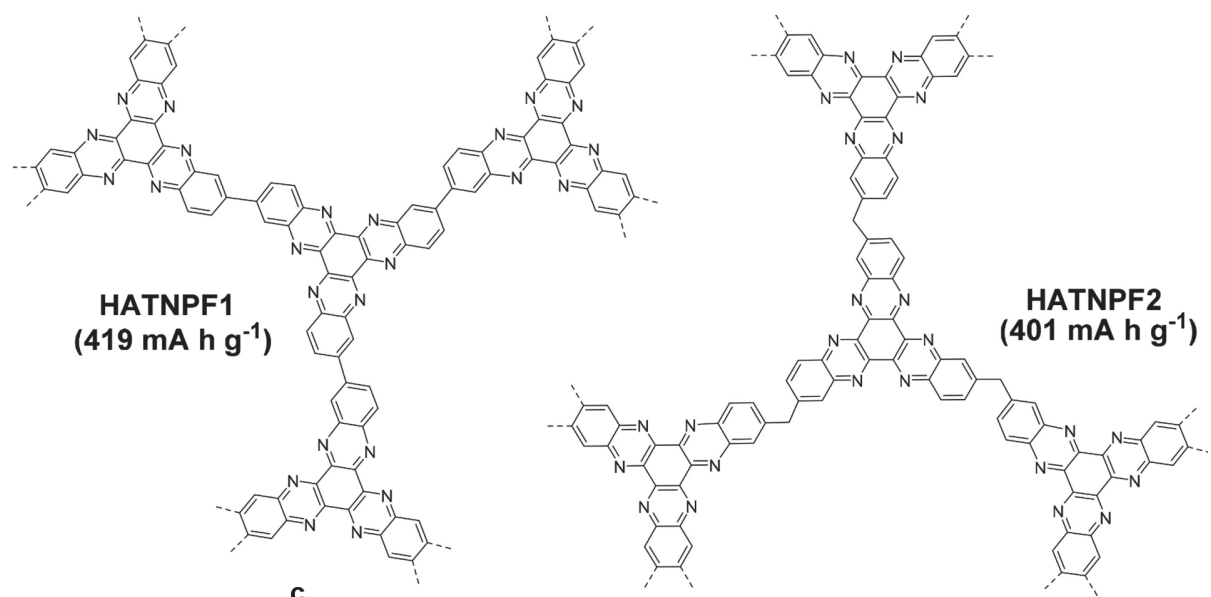
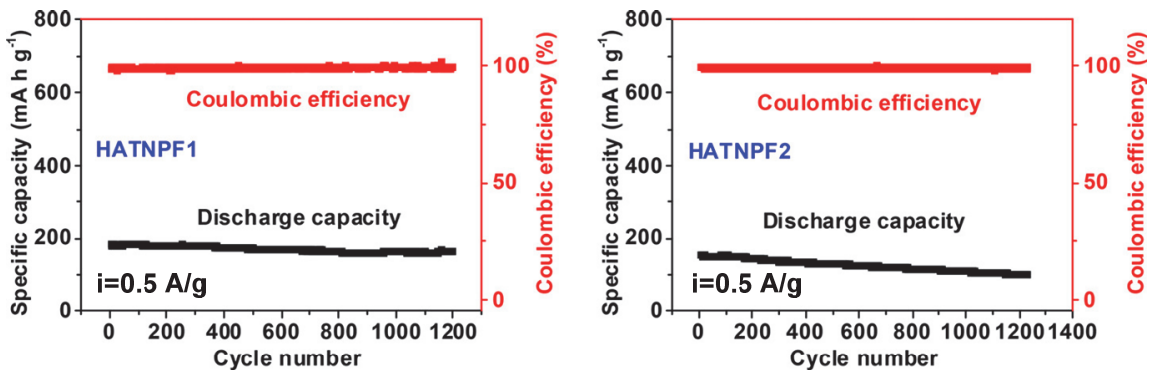

图 4 HATNPFs 的结构及它们的电化学性能表征 ${ }^{[26]}$

Figure 4 Structure of HATNPFs and their electrochemical performance characterization

(a) The structure and theoretical specific capacity of HATNPF1 and HATNPF2; (b) charge-discharge curve of HATNPF1; (c) comparison of cyclic stability of HATNPF1 and HATNPF2

比容量, 约为理论容量的 74\%(图 4b), 出色的长期循环 稳定性(1200 次循环后容量保持率为 $92 \%$ ) 和良好的速率 性能 $(2$ 与 $0.2 \mathrm{~A} / \mathrm{g}$ 的容量相比, 保持率为 $65 \%$ ), 由于具 有不同的共轭度、刚性和孔隙率, 与 HATNPF1 相比, 柔 性 HATNPF2 显示出相对较低的循环稳定性(1200 次循 环后的容量保留率为 $66 \%$ )(图 4c). HATNPF1 和 HATNPF2 正极的循环稳定性和实际容量均远高于 HATN-CMP 和当时报道的多孔聚合物.

虽然利用合成 HAT 衍生物/石墨烯复合电极和含 HAT 结构的有机多孔聚合物获得了具有长期稳定循环 性能的电极材料, 但是这些复合材料和聚合物仍然存在 电子和离子电导率低的问题. 理论上, 具有 HAT 结构的 框架材料应具有良好的电导率和可逆氧化还原反应位 点以及明确的孔道结构以实现有效的离子迁移 ${ }^{[27]}$. 电 导率通常与材料 $\mathrm{HOMO}$ (最高占据分子轨道) $-\mathrm{LUMO}$ (最 低未占据分子轨道)间隙有关, 较小的 HOMO-LUMO 间 隙会导致低电阻(高电导率) ${ }^{[28]}$. 基于上述原理, 张玉根 课题组 ${ }^{[29]}$ 设计并合成了一种新型的纳米多孔硫桥键连 六氮杂芸并荎聚合物 NSHATN(图 5a), 它具有狭窄的 HOMO-LUMO 间隙(图 5b)和 $1.26 \mathrm{~nm}$ 的均一纳米孔结 构. 众所周知, 在 Nyquist 图中, 高频范围的半圆与电荷 转移电阻有关, 而低频范围的直线与离子扩散控制的过
程相对应, NSHATN 在高频范围内显示直径为 $310 \sim 490$ $\Omega$ 的半圆形, 该值远低于 HATN(或取代的)单体(600 $1100 \Omega$ )和具有单键(520 $\Omega$ )或亚甲基桥连键(1100 $\Omega$ )连 接的 HATN 聚合物. 这样的低电阻值意味着 NSHATN 具有较高的导电率. 电化学测试(电压测试范围为 $1.5 \sim$ $4.0 \mathrm{~V}$; NSHATN, GO, PVDF 的比例为 $5: 4: 1$; 电解液 为 $\mathrm{LiCF}_{3} \mathrm{SO}_{3} / \mathrm{G} 4$ ) 结果表明, 在 $0.05 \mathrm{~A} / \mathrm{g}$ 下 NSHATN 正极 的初始放电容量为 $320 \mathrm{mAh} / \mathrm{g}$ (理论容量的 $86 \%$ ), 多次 循环后库伦效率接近 $100 \%$, 在 100 圈后容量保持在 234 $\mathrm{mAh} / \mathrm{g}$; 在 $8 \mathrm{~A} / \mathrm{g}$ 的高电流密度下 NSHATN 的容量为 $100 \mathrm{mAh} / \mathrm{g}$, 相当于在 $0.1 \mathrm{~A} / \mathrm{g}$ 的低电流密度下的容量的 $37 \%$, 当电流密度从 $8 \mathrm{~A} / \mathrm{g}$ 减小到 $0.1 \mathrm{~A} / \mathrm{g}$ 时, 容量回弹; $0.5 \mathrm{~A} / \mathrm{g}$ 下 1500 次循环后稳定在 $152 \mathrm{mAh} / \mathrm{g}$, 保留率为初 始容量的 $83 \%$. 从这些数据可以看出, NSHATN 阴极在 容量、速率能力和长期循环稳定性方面表现出良好电化 学性能, 循环过程中持续接近 $100 \%$ 的库伦效率表明, NSHATN 阴极实现了快速的充电和放电过程. 除此之 外, 该课题组 ${ }^{[30]}$ 经过密度泛函理论的计算, 对具有两个 HATN 单元和一种连接原子的模型单元进行了评估, 结 果表明, 经双氮键连接的 HATN 构建体的 LUMOHOMO 间隙最低. 以 $1,2,4,5$-苯四胺四盐酸盐为原料, 分两步合成了双氮连接的共轭 HATN 聚合物 $\mathrm{N}_{2}$ - 
HATN(图 5c). 端基的存在可能会削弱 $\mathrm{N}_{2}$-HATN 聚合物 与 $\mathrm{GO}$ 之间的相互作用, 增加离子和电子传输的阻力, 因而将 $\mathrm{N}^{2}$-HATN 退火处理得到 $\mathrm{N}^{2}-\mathrm{HATN} *$, 红外光谱和 紫外-可见吸收光谱显示退火处理除去了大多数末端官 能团, 且保留了核心共轭的 HATN 结构(图 $5 \mathrm{~d}$ ). 以 pRGO (GO 经以 $5{ }^{\circ} \mathrm{C} / \mathrm{min}$ 的升温速率在氮气下加热至 $350{ }^{\circ} \mathrm{C}$, 并保持 $4 \mathrm{~h}$ 制得)为导电剂, 得到具有富氮共轭 结构的 $\mathrm{N}^{2}-\mathrm{HATN} * / \mathrm{pRGO}$ 电极. 该电极在循环过程中出 现了容量异常快速升高的现象, 推测活性物质在循环过 程中被进一步活化或者聚合物、pRGO 和电解液之间形 成了新键或有其他相互作用.

除了在聚合物骨架设计上针对有机电极电导率低 的缺点来进行考虑外, 还可以通过有机聚合物与导电基 质的复合来提高有机电极的导电性. 2018 年, 冯新亮课 题组 ${ }^{[31]}$ 用 HATN-6CHO 分别和 1,4-二氨基苯、1,4-苯二 乙腈反应, 合成了共轭聚合物骨架 $2 \mathrm{D} C=\mathrm{N}$ HATN 和 2D CCP-HATN(图 6a), 并将其作为锂离子电池的可持 续有机正极进行了电化学行为的研究, 发现 $2 \mathrm{D}$ CCPHATN 的氧化还原特性优于 $2 \mathrm{D} \mathrm{C}=\mathrm{N}$ HATN. 该课题组 为了充分暴露氧化还原活性位点并增强电导率, 在溶剂 热反应过程中, 将 $2 \mathrm{D}$ CCP-HATN 直接原位生长在碳纳 米管 $(\mathrm{CNT})$ 表面, 命名为 2D CCP-HATN@CNT, 透射电 子显微镜(TEM)图像揭示了 2D CCP-HATN@CNT 的核壳结构, 平均厚度约为 $2.8 \mathrm{~nm}$, 相当于约 7 层的 $2 \mathrm{D}$ CCP-HATN 壳(图 6b). 由于高导电性的 CNT 提供了电子 传输途径, 所得的复合材料 2D CCP-HATN@CNT(具有 $50 \%$ 的 $\mathrm{CNT}$ ) 显示出 $0.4 \mathrm{~S} / \mathrm{cm}$ 的电导率, 比原始 $2 \mathrm{D}$

(a)

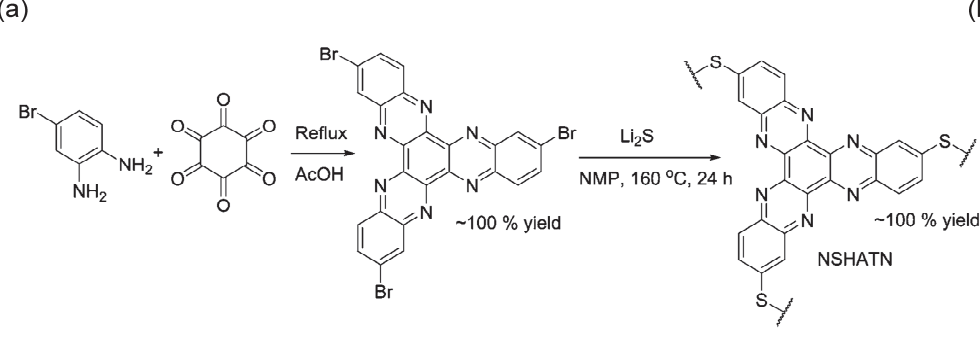

(c)
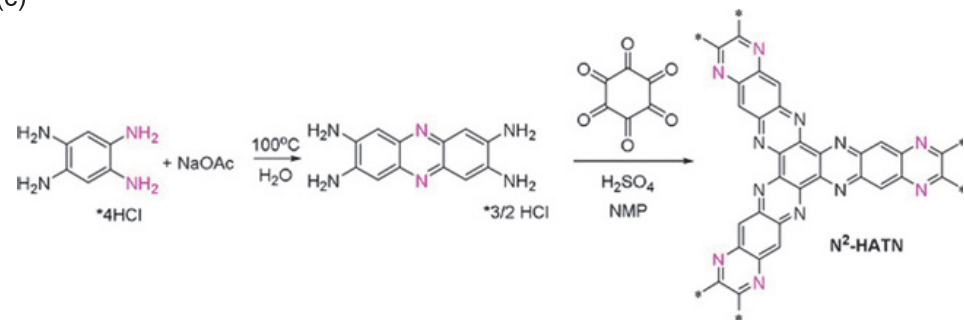

(d)
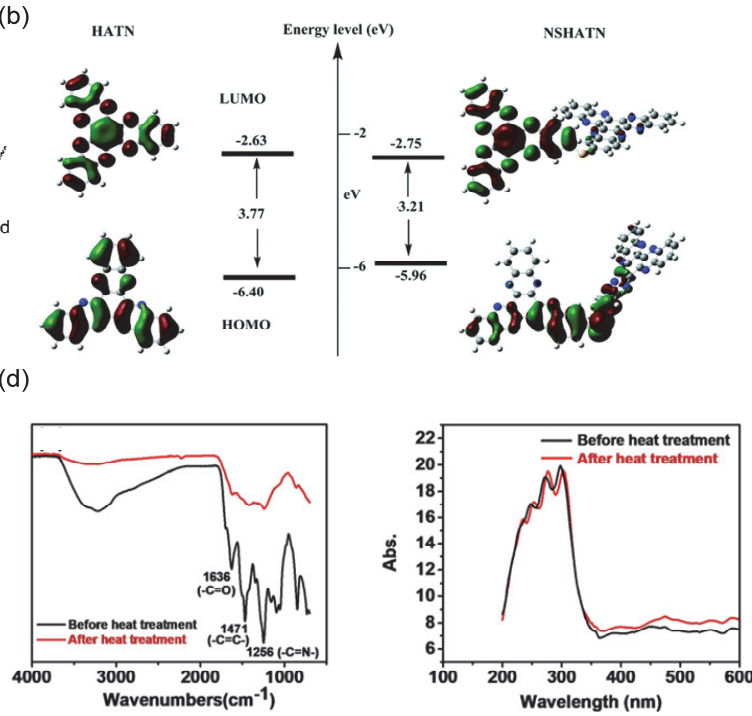

图 5 具备低 LUMO-HOMO 间隙的聚合物 NSHATN 和 $\mathrm{N}^{2}-\mathrm{HATN}{ }^{[29-30]}$

Figure 5 Polymers NSHATN and $\mathrm{N}^{2}$-HATN with low LUMO-HOMO gap

(a) The two-step synthesis of nanoporous sulfur-bridged hexaazatrinaphthylene polymer (NSHATN); (b) molecular structures and HOMO/LUMO energy levels of HATN and NSHATN; (c) nitrogen-linked HATN polymer ( $\mathrm{N}^{2}$-HATN) and its two-step synthesis approach; (d) FT-IR spectra and UV-vis spectra of $\mathrm{N}^{2}$-HATN polymer before and after heat treatment 


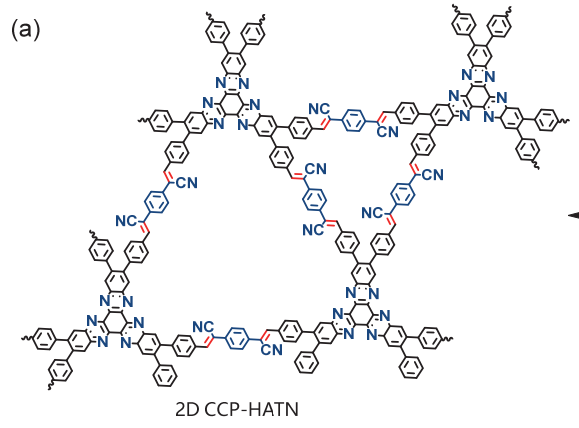

(b)

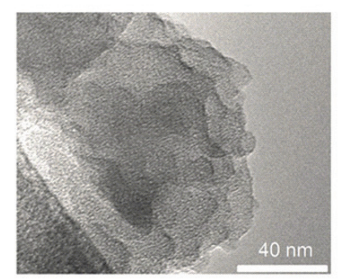

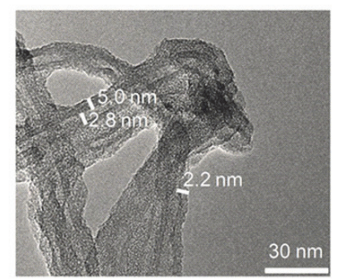

(c)

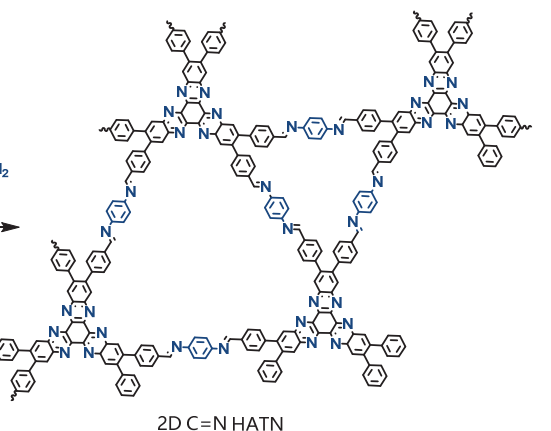

$2 D C=$ N HATN

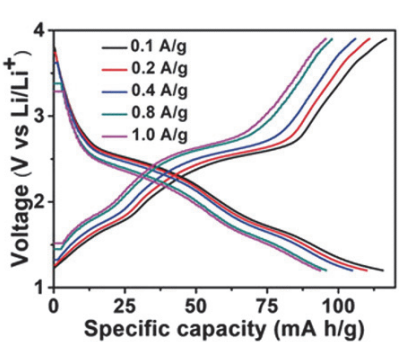

图 6 原位复合 $\mathrm{CNT}$ 制备高导电性的电极材料及电化学性能表征 ${ }^{[31]}$

Figure 6 In-situ composite CNT to prepare highly conductive electrode materials and their electrochemical performance characterization

(a) Scheme for the synthesis of 2D CCP-HATN and 2D C=N HATN from HATN-6CHO; (b) TEM images of 2D CCP-HATN and 2D CCP HATN@CNT; (c) CV curve of 2D C=N HATN, 2D CCP-HATN and 2D CCP-HATN@CNT, charge-discharge profiles of 2D CCP-HATN@CNT at different current densities

永胜课题组 ${ }^{[32]}$ 通过环己六酮 $(\mathrm{HKH})$ 和四氨基苯醌 (TABQ)的简单缩聚反应, 将 HAT 结构引入 COF 中, 合 成了具有高度 $\pi-\pi$ 共轭平面的共价有机框架材料 BQ1$\mathrm{COF}$ (图 7), 由吡嗪键连接的 BQ1-COF 具有相当稳定的 框架结构、清晰可见的晶格条纹和均一的孔道结构. 它 由最大的活性基团和最小的惰性单元组成, 每个重复分 子单元理论上具有 18 个电子的氧化还原, 理论比容量 高达 $773 \mathrm{mAh} / \mathrm{g}$. 当用作 LIB 的正极材料时, 电化学测 试(电压测试范围为 $1.2 \sim 3.5 \mathrm{~V}$; BQ1-COF, super $\mathrm{P}$, PVDF 比例为 $5: 4: 1$; 电解液为 LiTFSI-DOL/DME)表 明它具有 $502.4 \mathrm{mAh} / \mathrm{g}$ 的可逆比容量, 相当于每个重复 分子单元可转移 12 个电子, 能量密度可达 $1033 \mathrm{Wh} / \mathrm{kg}$; 稳定的框架结构具有出色的容量保持能力(在 $1.54 \mathrm{~A} / \mathrm{g}$ 下 1000 次循环后容量保持率为 $81 \%$ ), 在整个循环过程 中库伦效率接近 $100 \%$; 明确定义的孔道结构和扩展的 $\pi-\pi$ 共轭体系促进了 $\mathrm{Li}^{+}$的快速扩散和电子传输, 提供了 出色的速率性能(即使在 $7.73 \mathrm{~A} / \mathrm{g}$ 下也达到了 170.7 $\mathrm{mAh} / \mathrm{g}$ 的比容量), 远远优于以前的相关报道.

综上所述, 三亚吡嗪类材料作为锂离子电池正极材 料具有良好的电化学性能, 为此, 本课题组对三亚吡嗪 类材料于锂离子电池正极材料的应用也进行了探究. 我 们设计并运用离子热合成法, 使六氨基苯三盐酸盐 $(\mathrm{BH} \cdot 3 \mathrm{HCl})$ 分别与 2,7-二硝基萠-4,5,9,10-四酮(PTBH$\left.\mathrm{NO}_{2}\right)$ 、萠-4,5,9,10-四酮 $\left(\mathrm{PTBH}-\mathrm{NO}_{2}\right)$ 反应得到 $\mathrm{PTBH}-\mathrm{NO}_{2}$
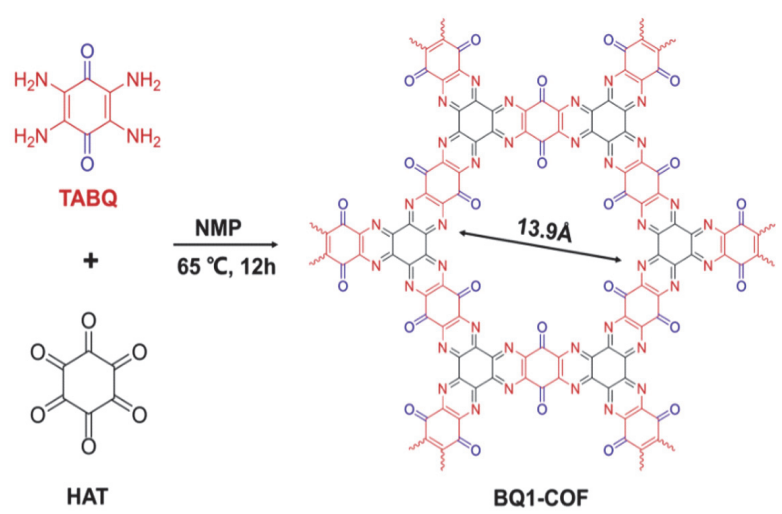

图 7 高理论比容量 $\mathrm{BQ1}-\mathrm{COF}$ 的设计合成 ${ }^{[32]}$

Figure 7 Scheme for the synthesis of BQ1-COF

和 PTBH, 将 HAT 结构引入到了聚合物中(图 8a). PTBH$\mathrm{NO}_{2}$ 和 PTBH 的高比表面积与开放的孔结构为电荷存储 提供了足够的界面, 使这些氧化还原位点能与锂离子充 分接触. $\mathrm{PTBH}-\mathrm{NO}_{2}$ 和 $\mathrm{PTBH}$ 作为锂离子电池正极材料 具有较好的倍率和循环性能. $\mathrm{PTBH}-\mathrm{NO}_{2}$ 作为锂离子电 池正极材料在电流密度为 $0.05 \mathrm{~A} / \mathrm{g}$ 时的放电容量为 314 $\mathrm{mAh} / \mathrm{g}$. 即使在 $3 \mathrm{~A} / \mathrm{g}$ 的高电流密度下, 也可以达到 148 $\mathrm{mAh} / \mathrm{g}$ 的高放电容量. 以电流密度为 $0.5 \mathrm{~A} / \mathrm{g}$ 循环 1000 次后容量保持率可达 $79 \%$, 循环期间库仑效率接近 $100 \%$. 由于硝基的存在提高了材料的锂吸附能力, 进 而提高了活性位点利用率, 这使得 $\mathrm{PTBH}-\mathrm{NO}_{2}$ 的容量高 
(a)

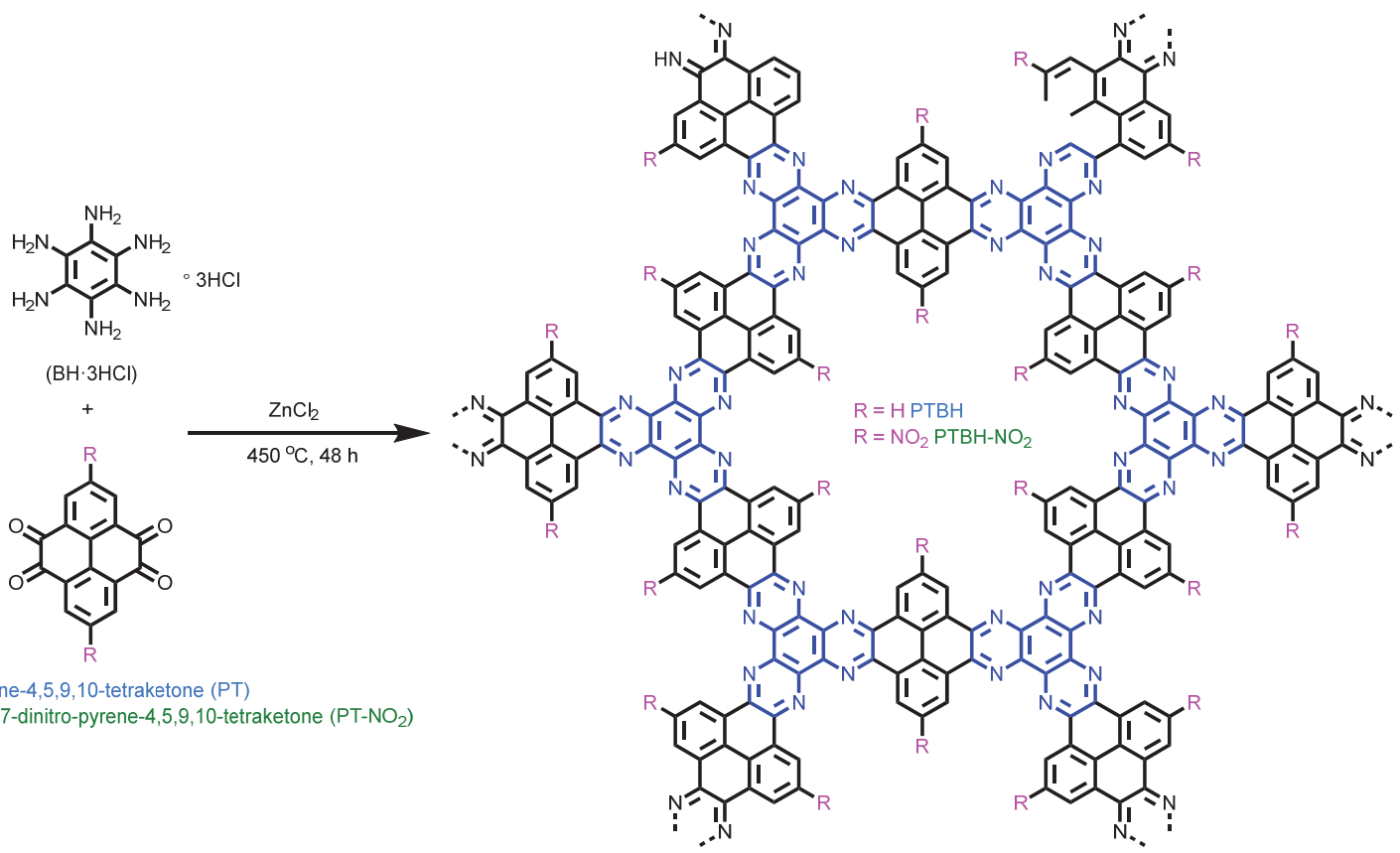

(b)
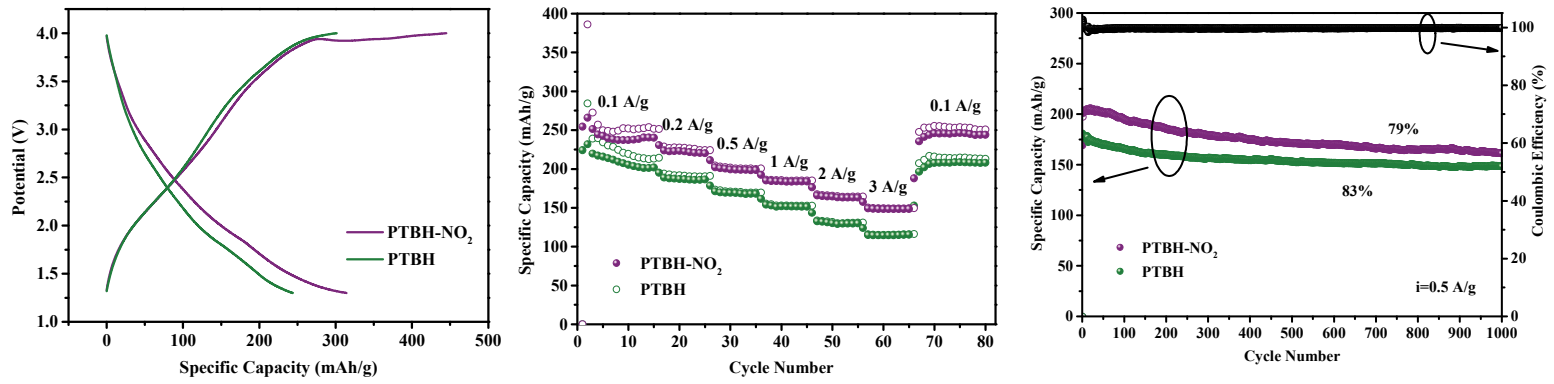

图 8 HAT 聚合物的合成及电化学表征 ${ }^{[33]}$

Figure 8 Synthesis and electrochemical characterization of HAT polymers

(a) Synthetic route of PTBH-NO 2 and PTBH; (b) the charge and discharge test, rate test and cycle stability test of PTBH-NO ${ }_{2}$ and PTBH in sequence

于 $\mathrm{PTBH}(\text { 图 8b) })^{[33]}$.

\subsection{HAT 衍生物在钠、镁、铝和锌离子电池方面的应}

用

虽然锂离子电池已经商业化, 但是锂离子电池在电 沉积过程中锂枝晶的生长会导致电池短路, 造成安全隐 患 ${ }^{[34]}$, 并且锂离子电池在大规模储能市场中的广泛应 用受到锂资源稀缺和分布不均的限制. 基于此, 需要寻 找性价比更高、更安全的电池电极. 研究人员发现钠的 性质类似于锂, 并且在地壳中储量丰富, 最重要的是钠 离子电池(NIB) 在储能领域中具有巨大潜力 ${ }^{[21]}$. 除了金 属钠之外, 镁和铝金属具有高体积能量密度、高丰度和 较低的反应性, 而且镁金属的无枝晶剥离/电镀工艺避 免了可充电电池内部短路带来的安全隐患, 并确保长期 的循环稳定性 ${ }^{[35]}$. 不仅如此, $\mathrm{Al}$ 和 $\mathrm{Mg}$ 的储存量分别占 地壳的 $7.73 \%$ 和 $2.5 \%$, 相对于 $\mathrm{Li}$ 而言是相当丰富的 $(\mathrm{Li}$ 占 $0.0017 \%)^{[28,36]}$. 因此多价可充电镁(RMB)和铝(RAB) 电池也引起了人们广泛的研究兴趣, 近几年 HAT 衍生
物在钠、镁和铝电池的正极材料研究上也有些探索.

2019 年王春生课题组 ${ }^{[37]}$ 制备了 HATN 的聚合物 PHATN, PHATN 与张玉根课题组制备的 HATNPF1 结构 相同, 不同的是 PHATN 被用作了 NIB、RMB 和 RAB 电池的通用阴极. 王春生课题组通过 XPS、FTIR、Raman 表征和 DFT 计算证明了氧化还原活性位点是基于 PHATN 中高度可逆的吡嗪基，他们将吡嗪衍生物用作 多价镁和铝电池的阴极，说明吡嗪基聚合物有望成为可 持续充电电池的通用阴极. 在 $\mathrm{NIBs}$ (电压测试范围为 $1.0 \sim 3.5$ V; PHATN, Ketjenblack, PVDF 比例为 $6: 3: 1$; 电解液为 $4 \mathrm{~mol} / \mathrm{L} \mathrm{NaPF} 6$-DME)中, PHATN 阴极具有快 速的反应动力学，良好的循环稳定性以及高能量密度： PHATN 在 $0.05 \mathrm{~A} / \mathrm{g}$ 下可产生 $220 \mathrm{mAh} / \mathrm{g}$ 的可逆容量, 随 着电流密度从 $0.05 \mathrm{~A} / \mathrm{g}$ 增加到 $2 \mathrm{~A} / \mathrm{g}$, 容量显示出从 220 $\mathrm{mAh} / \mathrm{g}$ 到 $164 \mathrm{mAh} / \mathrm{g}$ 的小幅下降, 即使在 4.8 和 $10 \mathrm{~A} / \mathrm{g}$ 的高电流密度下, PHATN 仍分别保持 138 和 $105 \mathrm{mAh} / \mathrm{g}$ 的可逆容量, 表明在钠插入和脱去过程中具有快速的反 应动力学; 在 $2 \mathrm{~A} / \mathrm{g}$ 下, 经过 10000 次循环后容量为 165 
$\mathrm{mAh} / \mathrm{g}$, 容量保持率为 $89.2 \%$, 即使在 $10 \mathrm{~A} / \mathrm{g}$ 的超高电 流密度下, PHATN 在 50000 次循环后仍保持 $100 \mathrm{mAh} / \mathrm{g}$ 的容量, 这种高容量传递和保留是 NIBs 聚合物及无机 阴极中最好结果. 高容量和卓越的速率性能有助于实现 高能量密度和功率密度, PHATN 在 $20000 \mathrm{~W} / \mathrm{kg}$ 的超高 功率密度下可提供 $210 \mathrm{Wh} / \mathrm{kg}$ 的能量密度, 在 NIB 有机 阴极中具备很高的竞争力. 此外, PHATN 在 RMBs 和 RABs 中也表现出稳定的循环性能和快速的反应动力 学. 在 RMBs 中, 电流密度为 $20 \mathrm{~mA} / \mathrm{g}$, 经过 200 次循环 后, PHATN 保留了 $110 \mathrm{mAh} / \mathrm{g}$ 的可逆容量; 在 RABs 中, 电流密度为 $50 \mathrm{~mA} / \mathrm{g}$, 经过 100 次循环后, PHATN 保留 了 $92 \mathrm{mAh} / \mathrm{g}$ 的可逆容量.

实现同时具有高容量和高倍率性能的钠离子电池 材料是一项挑战. 陈军课题组 ${ }^{[38]}$ 合成了与 BQ1-COF 结 构相同的 TQBQ-COF, 将其作为钠离子电池的正极材 料. 原位傅立叶变换红外(FT-IR)光谱和密度泛函理论 计算表明, 羰基 $(C=O)$ 和吡嗪环中的亚胺键 $(C=N)$ 是 活性位点, 并且每个 TQBQ-COF 重复单元可以存储十 二个 $\mathrm{Na}^{+}$离子, 包括 $\mathrm{TQBQ}-\mathrm{COF}$ 平面内的六个 $\mathrm{Na}^{+}$离子 和平面外的六个 $\mathrm{Na}^{+}$离子(图 9). 具有丰富氮原子和多 孔共轭结构的导电 TQBQ-COF 材料能够促进电子在大 量氧化还原位点之间的迁移和 $\mathrm{Na}^{+}$离子的扩散, 并且 $\mathrm{N}$ 原子的引入减小了 LUMO 和 HOMO 之间的能隙, 增强 了电子电导率(约 $10^{-9} \mathrm{~S} / \mathrm{cm}$ ) 和离子电导率(约 $10^{-4}$ $\mathrm{S} / \mathrm{cm}$ ). 良好的反应动力学、高多孔性和氮掺杂共轭结构 确保了 TQBQ-COF 电极具有高容量和高倍率的钠电池 性能. 电化学测试[电压测试范围为: $1.0 \sim 3.6 \mathrm{~V}$; TQBQCOF, super P, PVDF 的比例为 $5: 4: 1$; 电解液为 $\mathrm{NaPF}_{6}$-DEGDME(二乙二醇二甲醚)]表明: 在 $0.02 \mathrm{~A} / \mathrm{g}$ 的 电流密度下, TQBQ-COF 电极显示出 $452 \mathrm{mAh} / \mathrm{g}$ 的高可 逆容量, 100 圈之后稳定保持在 $352.3 \mathrm{mAh} / \mathrm{g}$; 在 10.0 $\mathrm{A} / \mathrm{g}$ 时显示出 $134.3 \mathrm{mAh} / \mathrm{g}$ 的高倍率容量; 并且具有稳 定框架结构的 TQBQ-COF 电极表现出出色的循环稳定 性(在 $1.0 \mathrm{~A} / \mathrm{g}$ 下 1000 次循环后容量保持率达 $96 \%$ ).
水系充电电池成本低，具有不易燃水基电解质，本 质上是安全的, 并且在很大程度上不依赖于复杂电池管 理系统的保护 [39]. 但基于 ZIB Zn ${ }^{2+}$ 配位原理的有机活性 材料的电子传导性差, 并且它们相应的放电产物通常易 溶解在 $\mathrm{H}_{2} \mathrm{O}$ 中, 因而它们通常具有差的速率性能和有限 的循环寿命 ${ }^{[16]} . \mathrm{H}^{+}$具有小的离子半径和低的相对原子质 量, 被认为是有吸引力的电荷载体 ${ }^{[40]}$, 因此, 基于 $\mathrm{H}^{+}$的 快速动力学的质子存储方式的中性水系锌离子电池 (ZIB) 被广泛关注. 具有亚氨键的 $\pi$ 共轭芳族化合物 HATN 是一种中性有机碱，具有通过配位反应螯合质子 的能力, 亚氨基部分 $\mathrm{N}$ 原子中的孤对电子确保 HATN 具 有高氧化活性.

基于这些原理, 牛志强课题组 ${ }^{[41]}$ 在温和的电解质 中开发了具有 $\mathrm{H}^{+}$插入作用的 $\mathrm{Zn} / \mathrm{HATN}$ 水系电池. $\mathrm{Zn} /$ HATN 系统通过配位/去配位反应显示出 $\mathrm{H}^{+}$的嵌入/脱 嵌，而不是常规的 $\mathrm{Zn}^{2+}$ 嵌入/脱嵌. 由于 $\mathrm{H}^{+}$嵌入/脱嵌的 快速动力学, $\mathrm{Zn} / \mathrm{HATN}$ 水性电池实现了高比容量和改善 的速率性能; HATN 的 $\pi$ 共轭芳香结构增强了 $\pi-\pi$ 分子间 的相互作用，从而抑制了 HATN 在水性电解质中的溶 解, 确保了 Zn/HATN 电池的长期循环寿命. 电化学测试 (电压测试范围为: $0.3 \sim 1.1 \mathrm{~V}$; HATN 纳米带, super P, PVDF 的比例为 $6: 3.5: 0.5$; 电解液: $2 \mathrm{~mol} / \mathrm{L} \mathrm{ZnSO}_{4}$ ) 结 果表明: Zn/HATN 电池在 $100 \mathrm{~mA} / \mathrm{g}$ 的电流密度下具有 $405 \mathrm{mAh} / \mathrm{g}$ 的初始放电容量, 接近其理论容量 419 $\mathrm{mAh} / \mathrm{g}$, 并高于以前报道的 ZIBs 有机电极材料, 即使在 $20 \mathrm{~A} / \mathrm{g}$ 的高电流密度下(充电/放电过程在 $44 \mathrm{~s}$ 内完成) 仍可显示 $123 \mathrm{mAh} / \mathrm{g}$ 的高容量, 在 $5 \mathrm{~A} / \mathrm{g}$ 的电流密度下 经过 5000 次循环后仍保持在 $140 \mathrm{mAh} / \mathrm{g}$ 左右, 相当于 93.3\%的高容量保持率.

上述 HAT 衍生物的电池性能的总结见表 1.

\section{HAT 衍生物在钙钛矿太阳能电池方面的应 用}

有机太阳能电池由于质轻、价廉、柔性, 受到人们

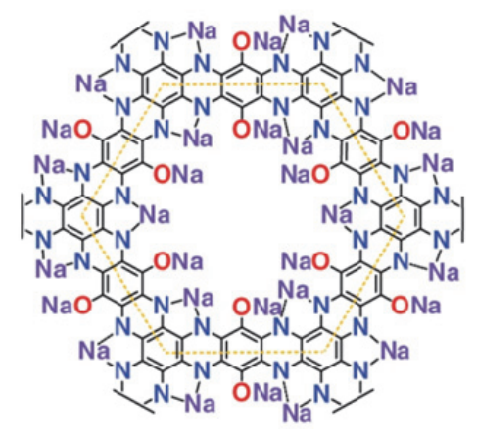

图 $9 \mathrm{TQBQ}-\mathrm{COF}$ 的化学结构和可能的电化学氧化还原机理 ${ }^{[38]}$

Figure 9 Chemical structure and possible electrochemical redox mechanism of TQBQ-COF 
表 1 基于 HAT 衍生物的有机电极的组成及电化学性能对比

Table 1 Comparison of the composition and electrochemical performance of organic electrodes based on HAT derivatives

\begin{tabular}{|c|c|c|c|c|c|}
\hline $\begin{array}{l}\text { 材料名称 } \\
\text { (电压窗口) }\end{array}$ & 电极组成及电解液 & $\begin{array}{c}\text { 原始和稳定时 } \\
\text { 容量及氧化还原利用率 }\end{array}$ & 循环稳定性 & 速率能力 & 文献 \\
\hline $\begin{array}{l}3 \mathrm{Q} \\
(1.2 \sim 3.9 \mathrm{~V})\end{array}$ & $\begin{array}{l}3 \mathrm{Q}: \text { graphene }: \text { PVDF } \\
(3: 6: 1) \\
1.0 \mathrm{~mol} / \mathrm{L} \\
\text { LiTFSI-DOL/DME }\end{array}$ & $\begin{array}{l}0.4 \mathrm{~mA} / \mathrm{g}: \\
395 \mathrm{mAh} / \mathrm{g} ; 94 \% \text { (1th) } \\
324 \mathrm{mAh} / \mathrm{g} ; 77 \% \text { (200th) }\end{array}$ & $\begin{array}{l}8 \mathrm{~A} / \mathrm{g}: \\
82 \%(2000) \\
80 \%(6000) \\
67 \%(10000)\end{array}$ & $\begin{array}{l}0.4 \mathrm{~A} / \mathrm{g}: 394 \mathrm{mAh} / \mathrm{g} \\
0.8 \mathrm{~A} / \mathrm{g}: 334 \mathrm{mAh} / \mathrm{g} \\
1.6 \mathrm{~A} / \mathrm{g}: 290 \mathrm{mAh} / \mathrm{g} \\
2.4 \mathrm{~A} / \mathrm{g}: 268 \mathrm{mAh} / \mathrm{g} \\
3.2 \mathrm{~A} / \mathrm{g}: 255 \mathrm{mAh} / \mathrm{g} \\
4 \mathrm{~A} / \mathrm{g}: 242 \mathrm{mAh} / \mathrm{g} \\
8 \mathrm{~A} / \mathrm{g}: 218 \mathrm{mAh} / \mathrm{g}\end{array}$ & [23] \\
\hline $\begin{array}{l}\mathrm{HATN} / \mathrm{GO} \\
(1.5 \sim 4.0 \mathrm{~V})\end{array}$ & $\begin{array}{l}\mathrm{HATN} / \mathrm{GO}: \mathrm{GO}: \mathrm{PVDF} \\
(5: 4: 1) \\
1 \mathrm{~mol} / \mathrm{L} \\
\mathrm{LiPF}_{6}-\mathrm{EC} / \mathrm{DEC}\end{array}$ & $\begin{array}{l}0.05 \mathrm{~A} / \mathrm{g}: \\
410 \mathrm{mAh} / \mathrm{g} ; 98 \%(1 \mathrm{th}) \\
226 \mathrm{mAh} / \mathrm{g} ; 54 \% \text { (90th) }\end{array}$ & $\begin{array}{l}0.5 \mathrm{~A} / \mathrm{g} \\
80 \%(2000)\end{array}$ & $\begin{array}{l}0.1 \mathrm{~A} / \mathrm{g}: 270 \mathrm{mAh} / \mathrm{g} \\
0.2 \mathrm{~A} / \mathrm{g}: 227 \mathrm{mAh} / \mathrm{g} \\
0.3 \mathrm{~A} / \mathrm{g}: 211 \mathrm{mAh} / \mathrm{g} \\
0.4 \mathrm{~A} / \mathrm{g}: 186 \mathrm{mAh} / \mathrm{g} \\
0.5 \mathrm{~A} / \mathrm{g}: 167 \mathrm{mAh} / \mathrm{g} \\
1 \mathrm{~A} / \mathrm{g}: 92 \mathrm{mAh} / \mathrm{g}\end{array}$ & {$[24]$} \\
\hline $\begin{array}{l}\text { HATNTA/GO } \\
(1.5 \sim 4.0 \mathrm{~V})\end{array}$ & $\begin{array}{l}\text { HATNTA/GO }: \text { GO }: \text { PVDF } \\
(5: 4: 1) \\
1 \mathrm{~mol} / \mathrm{L} \\
\text { LiPF }_{6}-\mathrm{EC} / \mathrm{DEC}\end{array}$ & $\begin{array}{l}0.05 \mathrm{~A} / \mathrm{g}: \\
226 \mathrm{mAh} / \mathrm{g} ; 73 \% \text { (1th) } \\
193 \mathrm{mAh} / \mathrm{g} ; 62 \% \text { (50th) }\end{array}$ & $\begin{array}{l}0.5 \mathrm{~A} / \mathrm{g} \\
86 \%(2000)\end{array}$ & $\begin{array}{l}0.1 \mathrm{~A} / \mathrm{g}: 215 \mathrm{mAh} / \mathrm{g} \\
0.2 \mathrm{~A} / \mathrm{g}: 193 \mathrm{mAh} / \mathrm{g} \\
0.3 \mathrm{~A} / \mathrm{g}: 183 \mathrm{mAh} / \mathrm{g} \\
0.4 \mathrm{~A} / \mathrm{g}: 170 \mathrm{mAh} / \mathrm{g} \\
0.5 \mathrm{~A} / \mathrm{g}: 133 \mathrm{mAh} / \mathrm{g} \\
1 \mathrm{~A} / \mathrm{g}: 114 \mathrm{mAh} / \mathrm{g}\end{array}$ & {$[24]$} \\
\hline $\begin{array}{l}\text { HATN-CMP } \\
(1.5 \sim 4.0 \mathrm{~V})\end{array}$ & $\begin{array}{l}\text { HATN-CMP : acetylene black: } \\
\text { PVDF } \\
(6: 3: 2) \\
1 \mathrm{~mol} / \mathrm{L} \\
\mathrm{LiPF}_{6}-\mathrm{EC} / \mathrm{DMC}\end{array}$ & $\begin{array}{l}0.1 \mathrm{~A} / \mathrm{g}: \\
147 \mathrm{mAh} / \mathrm{g} ; 71 \% \text { (1th) } \\
91 \mathrm{mAh} / \mathrm{g} ; 43 \% \text { (50th) }\end{array}$ & $\begin{array}{l}0.1 \mathrm{~A} / \mathrm{g}: \\
62 \%(50)\end{array}$ & $\begin{array}{l}0.1 \mathrm{~A} / \mathrm{g}: 147 \mathrm{mAh} / \mathrm{g} \\
0.5 \mathrm{~A} / \mathrm{g}: 65 \mathrm{mAh} / \mathrm{g}\end{array}$ & {$[25]$} \\
\hline $\begin{array}{l}\text { HATNPF1 } \\
(1.5 \sim 4.0 \mathrm{~V})\end{array}$ & $\begin{array}{l}\mathrm{HATNPF} 1: \mathrm{GO}: \mathrm{PVDF} \\
(4: 5: 1) \\
\mathrm{LiCF}_{3} \mathrm{SO}_{3} / \mathrm{G} 4\end{array}$ & $\begin{array}{l}0.05 \mathrm{~A} / \mathrm{g}: \\
309 \mathrm{mAh} / \mathrm{g} ; 74 \% \text { (1th) } \\
290 \mathrm{mAh} / \mathrm{g} ; 69 \% \text { (130th) }\end{array}$ & $\begin{array}{l}0.5 \mathrm{~A} / \mathrm{g} \\
92 \%(1200)\end{array}$ & $\begin{array}{l}0.1 \mathrm{~A} / \mathrm{g}: 267 \mathrm{mAh} / \mathrm{g} \\
2 \mathrm{~A} / \mathrm{g}: 174 \mathrm{mAh} / \mathrm{g}\end{array}$ & {$[26]$} \\
\hline $\begin{array}{l}\text { HATNPF2 } \\
(1.5 \sim 4.0 \mathrm{~V})\end{array}$ & $\begin{array}{l}\mathrm{HATNPF} 2: \mathrm{GO}: \mathrm{PVDF}(4: 5: 1) \\
\mathrm{LiCF}_{3} \mathrm{SO}_{3} / \mathrm{G} 4\end{array}$ & $\begin{array}{l}0.05 \mathrm{~A} / \mathrm{g}: \\
205 \mathrm{mAh} / \mathrm{g} ; 51 \% \text { (1th) } \\
115 \mathrm{mAh} / \mathrm{g} ; 29 \% \text { (130th) }\end{array}$ & $\begin{array}{l}0.5 \mathrm{~A} / \mathrm{g} \\
66 \%(1200)\end{array}$ & $\begin{array}{l}0.1 \mathrm{~A} / \mathrm{g}: 200 \mathrm{mAh} / \mathrm{g} \\
2 \mathrm{~A} / \mathrm{g}: 112 \mathrm{mAh} / \mathrm{g}\end{array}$ & {$[26]$} \\
\hline $\begin{array}{l}\text { NSHATN } \\
(1.5 \sim 4.0 \mathrm{~V})\end{array}$ & $\begin{array}{l}\text { NSHATN }: \text { GO }: \text { PVDF }(5: 4: 1) \\
\mathrm{LiCF}_{3} \mathrm{SO}_{3} / \mathrm{G} 4\end{array}$ & $\begin{array}{l}0.05 \mathrm{~A} / \mathrm{g}: \\
320 \mathrm{mAh} / \mathrm{g} ; 86 \% \text { (1th) } \\
234 \mathrm{mAh} / \mathrm{g} ; 63 \% \text { (100th) }\end{array}$ & $\begin{array}{l}0.5 \mathrm{~A} / \mathrm{g} \\
83 \%(1500)\end{array}$ & $\begin{array}{l}0.1 \mathrm{~A} / \mathrm{g}: 270 \mathrm{mAh} / \mathrm{g} \\
8 \mathrm{~A} / \mathrm{g}: 100 \mathrm{mAh} / \mathrm{g}\end{array}$ & [29] \\
\hline $\begin{array}{l}\text { 2D CCP-HATN } \\
@ \mathrm{CNT} \\
(1.2 \sim 3.9 \mathrm{~V})\end{array}$ & $\begin{array}{l}\text { 2D CCP-HATN } \\
\text { @CNT }: \text { Super P }: \operatorname{Alg}(8: 1: 1) \\
1 \mathrm{~mol} / \mathrm{L} \\
\text { LiTFSI-DOL/DME }\end{array}$ & $\begin{array}{l}0.1 \mathrm{~A} / \mathrm{g}: \\
116 \mathrm{mAh} / \mathrm{g} ; 73 \%(1 \mathrm{th}) \\
114 \mathrm{mAh} / \mathrm{g} ; 73 \%(50 \mathrm{th})\end{array}$ & $\begin{array}{l}0.5 \mathrm{~A} / \mathrm{g} \\
91.2 \%(1000)\end{array}$ & $\begin{array}{l}0.1 \mathrm{~A} / \mathrm{g}: 116 \mathrm{mAh} / \mathrm{g} \\
1 \mathrm{~A} / \mathrm{g}: 94 \mathrm{mAh} / \mathrm{g}\end{array}$ & {$[31]$} \\
\hline $\begin{array}{l}\text { BQ1-COF } \\
(1.2 \sim 3.5 \mathrm{~V})\end{array}$ & $\begin{array}{l}\text { BQ1-COF }: \text { Super P }: \text { PVDF } \\
(5: 4: 1) \\
1 \mathrm{~mol} / \mathrm{L} \\
\text { LiTFSI-DOL/DME }\end{array}$ & $\begin{array}{l}0.039 \mathrm{~A} / \mathrm{g} \\
502 \mathrm{mAh} / \mathrm{g} ; 65 \%(2 \mathrm{th})\end{array}$ & $\begin{array}{l}1.54 \mathrm{~A} / \mathrm{g} \\
81 \%(1000)\end{array}$ & $\begin{array}{l}0.039 \mathrm{~A} / \mathrm{g}: 502 \mathrm{mAh} / \mathrm{g} \\
7.73 \mathrm{~A} / \mathrm{g}: 171 \mathrm{mAh} / \mathrm{g}\end{array}$ & {$[32]$} \\
\hline $\begin{array}{l}\operatorname{PHATN}(\mathrm{Na}) \\
(1.0 \sim 3.5 \mathrm{~V})\end{array}$ & $\begin{array}{l}\text { PHATN }: \text { Ketjenblack }: \text { PVDF } \\
(6: 3: 1) \\
4 \text { or } 1 \mathrm{~mol} / \mathrm{L} \mathrm{NaPF}_{6}-\mathrm{DME}\end{array}$ & $\begin{array}{l}0.05 \mathrm{~A} / \mathrm{g}: \\
220 \mathrm{mAh} / \mathrm{g}(2 \mathrm{th}) \\
205 \mathrm{mAh} / \mathrm{g}(100 \mathrm{th})\end{array}$ & $\begin{array}{l}2 \mathrm{~A} / \mathrm{g} \\
89.2 \%(10000) \\
10 \mathrm{~A} / \mathrm{g} \\
83.8 \%(50000)\end{array}$ & $\begin{array}{l}0.05 \mathrm{~A} / \mathrm{g}: 220 \mathrm{mAh} / \mathrm{g} \\
2 \mathrm{~A} / \mathrm{g}: 164 \mathrm{mAh} / \mathrm{g} \\
4.8 \mathrm{~A} / \mathrm{g}: 138 \mathrm{mAh} / \mathrm{g} \\
10 \mathrm{~A} / \mathrm{g}: 105 \mathrm{mAh} / \mathrm{g}\end{array}$ & {$[37]$} \\
\hline $\begin{array}{l}\text { PHATN(Mg) } \\
(0.5 \sim 2.3 \mathrm{~V})\end{array}$ & $\begin{array}{l}\text { PHATN: Ketjenblack: PTFE } \\
(6: 3: 1)\end{array}$ & $\begin{array}{l}0.02 \mathrm{~A} / \mathrm{g}: \\
146 \mathrm{mAh} / \mathrm{g}(1 \mathrm{th}) \\
110 \mathrm{mAh} / \mathrm{g}(200 \mathrm{th})\end{array}$ & $\begin{array}{l}0.02 \mathrm{~A} / \mathrm{g} \\
89 \%(200)\end{array}$ & $\begin{array}{l}0.02 \mathrm{~A} / \mathrm{g}: 125 \mathrm{mAh} / \mathrm{g} \\
0.2 \mathrm{~A} / \mathrm{g}: 60 \mathrm{mAh} / \mathrm{g}\end{array}$ & {$[37]$} \\
\hline
\end{tabular}




\begin{tabular}{|c|c|c|c|c|c|}
\hline $\begin{array}{l}\text { 材料名称 } \\
\text { (电压窗口) }\end{array}$ & 电极组成及电解液 & $\begin{array}{c}\text { 原始和稳定时 } \\
\text { 容量及氧化还原利用率 }\end{array}$ & 循环稳定性 & 速率能力 & 文献 \\
\hline $\begin{array}{l}\operatorname{PHATN}(\mathrm{Al}) \\
(0.2 \sim 1.2 \mathrm{~V})\end{array}$ & $\begin{array}{l}\text { PHATN : Ketjenblack: PTFE } \\
(6: 3: 1) \\
\mathrm{AlCl}_{3}-[\mathrm{BMIm}] \mathrm{Cl} \text { 离子液体 }\end{array}$ & $\begin{array}{l}0.05 \mathrm{~A} / \mathrm{g}: \\
145 \mathrm{mAh} / \mathrm{g}(1 \mathrm{th}) \\
92 \mathrm{mAh} / \mathrm{g}(100 \mathrm{th})\end{array}$ & $\begin{array}{l}0.05 \mathrm{~A} / \mathrm{g} \\
87 \%(100)\end{array}$ & & [37] \\
\hline $\begin{array}{l}\text { Zn-HATN } \\
(0.2 \sim 1.2 \mathrm{~V})\end{array}$ & $\begin{array}{l}\text { Zn-HATN }: \text { Super P }: \text { PVDF } \\
(6: 3.5: 0.5) \\
2 \mathrm{~mol} / \mathrm{L} \mathrm{ZnSO}_{4}\end{array}$ & $\begin{array}{l}0.1 \mathrm{~A} / \mathrm{g}: \\
405 \mathrm{mAh} / \mathrm{g} ; 96 \% \text { (1th) } \\
320 \mathrm{mAh} / \mathrm{g} ; 76 \% \text { (150th) }\end{array}$ & $\begin{array}{l}5 \mathrm{~A} / \mathrm{g} \\
93.3 \%(5000)\end{array}$ & $\begin{array}{l}0.1 \mathrm{~A} / \mathrm{g}: 370 \mathrm{mAh} / \mathrm{g} \\
20 \mathrm{~A} / \mathrm{g}: 123 \mathrm{mAh} / \mathrm{g}\end{array}$ & {$[41]$} \\
\hline $\begin{array}{l}\text { TQBQ-COF }(\mathrm{Na}) \\
(1.0 \sim 3.6 \mathrm{~V})\end{array}$ & $\begin{array}{l}\text { TQBQ-COF }: \text { Super P }: \text { PVDF } \\
(5: 4: 1) \\
1.0 \mathrm{~mol} / \mathrm{L} \\
\mathrm{NaPF}_{6}-\mathrm{DEGDME}\end{array}$ & $\begin{array}{l}0.02 \mathrm{~A} / \mathrm{g}: \\
452 \mathrm{mAh} / \mathrm{g} ; 88 \% \text { (1th) } \\
352 \mathrm{mAh} / \mathrm{g} ; 68 \% \text { (100th) }\end{array}$ & $\begin{array}{l}1 \mathrm{~A} / \mathrm{g} \\
96 \%(1000)\end{array}$ & $\begin{array}{l}0.3 \mathrm{~A} / \mathrm{g}: 278 \mathrm{mAh} / \mathrm{g} \\
1.0 \mathrm{~A} / \mathrm{g}: 234 \mathrm{mAh} / \mathrm{g} \\
5.0 \mathrm{~A} / \mathrm{g}: 181 \mathrm{mAh} / \mathrm{g} \\
10 \mathrm{~A} / \mathrm{g}: 134 \mathrm{mAh} / \mathrm{g}\end{array}$ & [38] \\
\hline $\begin{array}{l}\text { HAT550@ZTC } \\
\text { (Na 离子电容器) } \\
(0.5 \sim 2.5 \mathrm{~V})\end{array}$ & $\begin{array}{l}\text { HAT550@ZTC：Super P：羧甲基 } \\
\text { 纤维素钠/聚丙烯酸 }(8: 1: 1) \\
1.0 \mathrm{~mol} / \mathrm{L} \mathrm{NaClO}_{4}-\mathrm{EC} / \mathrm{PC} / \mathrm{FEC}\end{array}$ & $\begin{array}{l}0.1 \mathrm{~A} / \mathrm{g}: \\
448 \mathrm{mAh} / \mathrm{g} ;(1 \mathrm{th}) \\
343 \mathrm{mAh} / \mathrm{g} ;(40 \mathrm{th})\end{array}$ & $\begin{array}{l}0.5 \mathrm{~A} / \mathrm{g} \\
90 \%(1300)\end{array}$ & $\begin{array}{l}0.1 \mathrm{~A} / \mathrm{g}: 343 \mathrm{mAh} / \mathrm{g} \\
20 \mathrm{~A} / \mathrm{g}: 124 \mathrm{mAh} / \mathrm{g}\end{array}$ & {$[48]$} \\
\hline
\end{tabular}

的广泛关注 ${ }^{[42]}$. HATN 衍生物具有廉价、迁移率高以及 带隙宽等特点, 是一种有潜力的电子传输材料 [11a,43]. 2016 年, Jen 课题组 ${ }^{[44]}$ 为了改善 HATN 衍生物的溶液加 工性能并微调其能级, 制备了一系列具有不同长度和不 同硫氧化状态(硫醚、亚砜和砜)的烷基磺胺基链的 HATN 衍生物. 使用 HATNA-F3(此文章中将 HATN 命名 为 HATNA) 衍生物 HATNASOC7-CS(图 10a) 作为电子传 输材料(ETM)制造的倒置平面结构(p-i-n 型)钙钛矿太阳 能电池(PSCs)显示出 $17.6 \%$ 的光电能量转换效率(PCE), 而滞后可以忽略不计. 这是使用非富勒烯有机 ETM 的 第一个实例, 其性能可与基于 $\operatorname{PCBM}$ (苯基-C61-丁酸甲 酯)的倒置钙钛矿太阳能电池相謧美, 这表明 HAT 衍生 物在改善 PCE 方面显示出巨大的潜力.

2020 年, 李泽生课题组在 Jen 课题组工作基础上设 计了一系列 HAT 衍生物, 旨在改善钙钛矿/ETM 界面的 电子提取并增加电子迁移率. 李泽生等 ${ }^{[45]}$ 通过将甲氧 基苯基、酰亚胺和菜基团引入 HAT 骨架, 合成了三种新
的 ETM (HAT-1、HAT-2 和 HAT-3)(图 10b). 李泽生课题 组通过理论计算证明 HAT-1、HAT-2 和 HAT-3 的电子迁 移率分别是 HATNASOC7- $\mathrm{C}_{\mathrm{S}}$ 的 2.98、3.79 和 13.21 倍, 这是因为萗的引入降低了重组能，而甲氧基苯基和酰亚 胺基团的引入通过 $\mathrm{C} \cdots \mathrm{C}$ 和 $\mathrm{H} \cdots \mathrm{O}$ 的相互作用增加了分 子间相互作用. $\mathrm{MAPbI}_{3} / \mathrm{ETMs}$ 界面研究表明, 甲氧基苯 基和酰亚胺基团的氧原子与 $\mathrm{MAPbI}_{3}$ 的铅原子形成 $\mathrm{O} \cdots$ $\mathrm{Pb}$ 界面相互作用, 增加了吸附能, 改变了投影态密度 (PDOS)结构, 改变了界面的空间分布. 因此新设计的 ETM 可以有效地提高电子提取率，从而提高短路电流 并具有更好的整体性能. 因此, 这三种 HAT 衍生物作为 高效倒置钙钛矿太阳能电池的 ETM 是相当有前途的.

\section{3 电容器方面的应用}

超级电容器既具有电容器快速充放电的特性, 同时 又具有电池的储能特性. 为满足车辆和便携式电子设备 供电的需求, 超级电容器的研究备受青睐 ${ }^{[46]}$, HAT 衍生 (a)

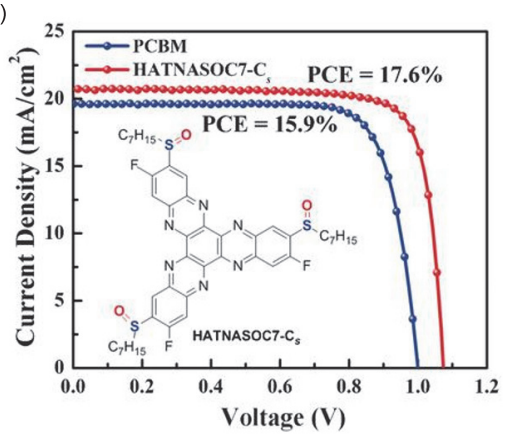

(b)

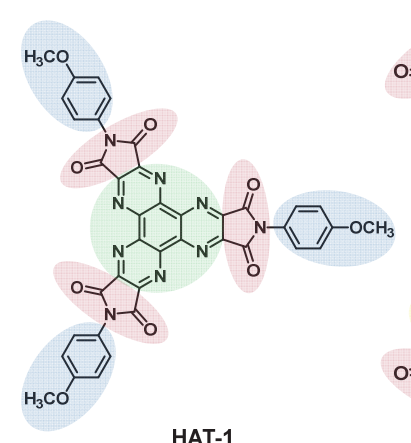

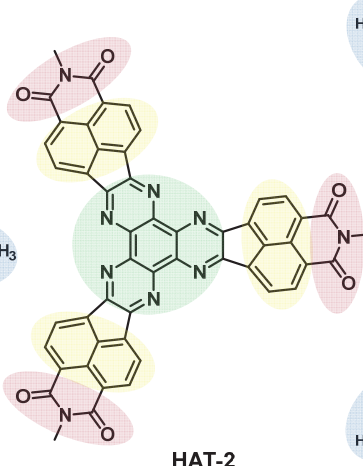

HAT-2

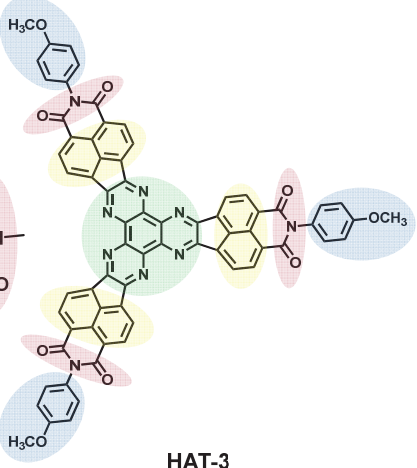

HAT-3

图 10 HAT 衍生物用于钙钛矿太阳能电池 ${ }^{[44-45]}$

Figure 10 HAT derivatives are used in perovskite solar cells

(a) The structure of HATNASOC7-C $\mathrm{C}_{\mathrm{S}}$, the J-V curve comparison chart of PVSC prepared with ETM and their PCE; (b) three HAT derivative structures prepared by Li's group 
物也可以作为超级电容器材料. 在 2011 年, 江东林课题 组 ${ }^{[47]}$ 通过苯四胺与环己六酮的离子热反应构建了具有 内置氮杂单元的多孔框架 Aza-CMP(图 11). 骨架中的氮 杂单元, 可与电解质阳离子发生偶极相互作用, 并在孔 壁上积聚质子; Aza-CMP 具有最佳尺寸的固有微孔，允 许离子在充放电过程中快速运动; Aza-CMP 的高表面积 为孔中形成静电电荷分离层提供了较大的界面. 这些特 征协同作用下, 实现了出色的能量存储和电源供应能 力. 电化学研究证明 Aza-CMP 具有大电容, 高能量和高 功率密度, 并且能够以优异的循环寿命进行重复的能量 存储和电源供应.
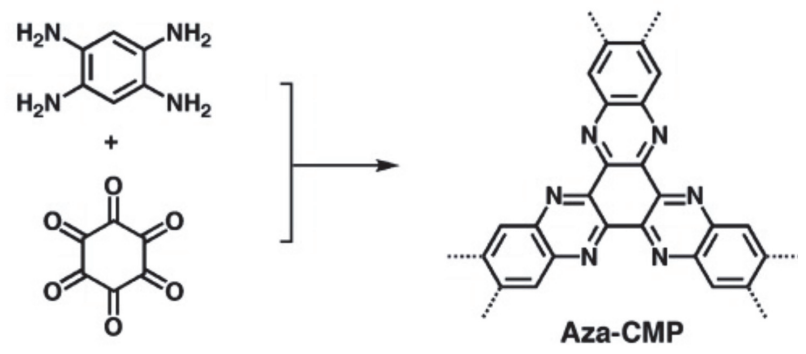

图 11 Aza-CMP 的结构及合成 ${ }^{[47]}$

Figure 11 Structure and synthesis of Aza-CMP

为了提高钠离子电容器的性能, 2019 年, Antonietti 课题组 ${ }^{[48]}$ 通过将掺杂氮的碳纳米颗粒和中孔碳结合合 成了纳米复合材料, 实现了较高的电导率和钠存储容 量. 该课题组首先使用 $\mathrm{ZnO}$ 纳米颗粒 $(20 \mathrm{~nm})$ 作为定义 明确的中孔硬模板来制备 ZTC $(\mathrm{ZnO}$ 模板碳, 表示为 “ZTC”), 即导电基质, 以容纳六氮杂三苯并六碳氰 (HAT-CN) 的富氮碳纳米颗粒, 制得嵌入到导电介孔碳 基体中的纳米复合材料 HAT550@ZTC. 该复合材料因 存在 HAT550 组分使氮元素质量分数高达 $26 \%$. HAT550 纳米颗粒(钠存储)和导电 ZTC 基质(快速电子存储和传 输)与复杂的纳米体系结构(快速离子传输)的集成有助 于充分利用每种成分，而存储位置足够接近也可以避免 更大的极化效应. HAT550@ZTC 复合材料具有出色的 钠存储性能, 电化学测试(电压测试范围为: $0.5 \sim 2.5 \mathrm{~V}$; HAT550@ZTC, super P, 羧甲基纤维素钠/聚丙烯酸的比 例为 $8: 1: 1$; 电解液为 $\mathrm{NaClO}_{4}-\mathrm{EC} / \mathrm{PC}($ 碳酸丙烯 酯)/FEC(氟代碳酸乙烯酯))表明: HAT550@ZTC 在 0.1 $\mathrm{A} / \mathrm{g}$ 时可逆容量为 $343 \mathrm{mAh} / \mathrm{g}$, 在 $20 \mathrm{~A} / \mathrm{g}$ 下稳定时的容 量为 $124 \mathrm{mAh} / \mathrm{g}$; 在 $0.5 \mathrm{~A} / \mathrm{g}$ 下 1300 次循环后容量保持 率达到 90\%. 以 HAT550@ZTC 为阳极的钠离子电容器 (NIC) 全电池装置在 $1.0 \sim 4.0 \mathrm{~V}$ 的电压范围内, 提供了 显著的能量和功率密度 $(0.1 \mathrm{kw} / \mathrm{kg}$ 时为 $61 \mathrm{Wh} / \mathrm{kg}, 24$ $\mathrm{kw} / \mathrm{kg}$ 时仍保持 $12 \mathrm{Wh} / \mathrm{kg}$ ).

\section{4 作为电池的保护材料}

富镍层状金属氧化物具有超过 $200 \mathrm{mAh} / \mathrm{g}$ 的高比容 量且对钴的依赖性低, 被选为电动汽车锂离子电池正极 材料，但过渡金属与锂离子之间发生阳离子混合会导致 较差的循环寿命和热稳定性 [49], 为了能够广泛而可靠 地利用这些材料, 2020 年, Choi 课题组 ${ }^{[50]}$ 通过环已六酮 和苯四胺缩合制备了导电性良好的二维共价有机框架 Pyr-2D, 并将其作为保护涂层材料应用于富镍的 $\mathrm{LiNi}_{0.8} \mathrm{Co}_{0.1} \mathrm{Mn}_{0.1} \mathrm{O}_{2}$ (NCM811) 电极表面. Pyr-2D 中具有 大量的 HAT 单元, HAT-COF 的二维平面形态沿着 NCM 颗粒的表面对齐, 吡嗪成为涂覆 NCM 材料的有用成份; 并且由于二维框架中共轭原子构型的中心作用使 Pyr-2D 具有良好的导电率. 使用 Pyr-2D 涂层后, NCM811 电极具有改善的速率能力、良好循环性能和高 温耐受性. 涂有 Pyr-2D 的电极的电化学测试结果表明, Pyr-2D 具有有效的保护作用, 可防止不良的副反应和避 免氢氟酸(HF)侵蚀.

\section{5 总结与展望}

发展可再生能源为解决当前化石能源短缺提供了 思路. 但是由于可再生能源的间歇性特征, 若实现广泛 利用, 迫切需要发展电化学储能技术. HAT 分子结构作 为金属离子电池电极不仅具有多个氧化活性位点, 理论 上可提供高比容量, 而且它的 $\pi-\pi$ 共轭平面有利于电子 和离子的快速传输, 从而增强材料导电性. 本文主要综 述了 HAT 衍生物在锂、钠、铝、镁、锌离子电池、钙 钛矿电池、电容器和电池表面保护材料等电化学储能方 面的研究进展. 目前, HAT 二维聚合物由于层与层之间 的堆积作用部分掩盖了材料活性位点，其实际容量未能 达到理论水平, HAT 衍生物作为电化学储能材料仍有探 索空间, 未来在 HAT 结构氧化还原位点的充分利用方 面还需探究. 但由于 HAT 具有出色的电子缺陷性质和 独特的拓扑结构, HAT 衍生物在电化学储能领域定能大 放异彩.

\section{References}

[1] Nasielski-Hinkens, R.; Benedek-Vamos, M.; Maetens, D.; Nasielski, J. J. Organomet. Chem. 1981, 217, 179.

[2] (a) Deng, H. L.; Luo, X. S.; Li, Z. H.; Zhao, J. Y.; Huang, M. H. Chin. J. Org. Chem. 2021, 41, 624 (in Chinese).

(邓汉林, 罗贤升, 李志华, 赵江颖, 黄木华, 有机化学, 2021, 41, 624.)

(b) Pang, C. M.; Luo, S. H.; Hao, Z. F.; Gao, J.; Huang, Z. H.; Yu, J. H.; Yu, S. M.; Wang, Z. Y. Chin. J. Org. Chem. 2018, 38, 2606 (in Chinese).

(庞楚明, 罗时荷, 郝志峰，高健，黄召昊，余家海，余思敏，汪 朝阳，有机化学, 2018, 38, 2606.)

[3] (a) Kitagawa, S.; Masaoka, S. Coord. Chem. Rev. 2003, 246, 73. (b) Segura, J. L.; Juárez, R.; Ramos, M.; Seoane, C. Chem. Soc. Rev. 2015, 44, 6850 
(c) Yan, X.-Y.; Lin, M.-D.; Zheng, S.-T.; Zhan, T.-G.; Zhang, X.; Zhang, K.-D.; Zhao, X. Tetrahedron Lett. 2018, 59, 592.

[4] (a) Liu, R.; von Malotki, C.; Arnold, L.; Koshino, N.; Higashimura, H.; Baumgarten, M.; Müllen, K. J. Am. Chem. Soc. 2011, 133, 10372.

(b) Ibáñez, S.; Poyatos, M.; Peris, E. Chem. Commun. 2017, 53, 3733.

[5] Ramimoghadam, D.; Gray, E. M.; Webb, C. J. Int. J. Hydrogen Energy 2016, 41, 16944.

[6] (a) Lee, J.-S. M.; Cooper, A. I. Chem. Rev. 2020, 120, 2171.

(b) Xu, Y.; Jin, S.; Xu, H.; Nagai, A.; Jiang, D. Chem. Soc. Rev. 2013, $42,8012$.

[7] (a) Hisaki, I.; Suzuki, Y.; Gomez, E.; Cohen, B.; Tohnai, N.; Douhal, A. Angew. Chem., Int. Ed. 2018, 57, 12650.

(b) Hisaki, I.; Suzuki, Y.; Gomez, E.; Ji, Q.; Tohnai, N.; Nakamura, T.; Douhal, A. J. Am. Chem. Soc. 2019, 141, 2111.

[8] (a) Côté, A. P.; Benin, A. I.; Ockwig, N. W.; Keeffe, M.; Matzger, A. J.; Yaghi, O. M. Science 2005, 310, 1166.

(b) Ding, S.-Y.; Wang, W. Chem. Soc. Rev. 2013, 42, 548

(c) Huang, Z.; Xu, Q.; Hu, X. Chin. Chem. Lett. 2020, 31, 2495.

[9] Meng, Z.; Aykanat, A.; Mirica, K. A. Chem. Mater. 2019, 31, 819.

[10] (a) Yuan, F.; Li, J.; Namuangruk, S.; Kungwan, N.; Guo, J.; Wang, C. Chem. Mater. 2017, 29, 3971.

(b) Tahir, N.; Wang, G.; Onyshchenko, I.; De Geyter, N.; Leus, K.; Morent, R.; Van Der Voort, P. J. Catal. 2019, 375, 242.

(c) Huang, H.; Zhao, Y.; Bai, Y.; Li, F.; Zhang, Y.; Chen, Y. Adv. Sci. 2020, 7, 2000012.

(d) Xiao, R.; Tobin, J. M.; Zha, M.; Hou, Y.-L.; He, J.; Vilela, F.; Xu, Z. J. Mater. Chem. A 2017, 5, 20180.

(e) Bai, C.; Wang, H.; Ning, F.; Fu, J.; Wei, J.; Lu, G.; Shen, Y.; Zhou, X. Chem CatChem 2020, 12, 4024.

[11] (a) Liu, X.-Y.; Usui, T.; Hanna, J. Chem.-Eur. J. 2014, 20, 14207. (b) Mahmood, J.; Lee, E. K.; Jung, M.; Shin, D.; Jeon, I.-Y.; Jung, S.-M.; Choi, H.-J.; Seo, J.-M.; Bae, S.-Y.; Sohn, S.-D.; Park, N.; Oh, J. H.; Shin, H.-J.; Baek, J.-B. Nat. Commun. 2015, 6, 6486.

[12] Gould, C. A.; Darago, L. E.; Gonzalez, M. I.; Demir, S.; Long, J. R. Angew. Chem., Int. Ed. 2017, 56, 10103.

[13] Walczak, R.; Kurpil, B.; Savateev, A.; Heil, T.; Schmidt, J.; Qin, Q.; Antonietti, M.; Oschatz, M. Angew. Chem., Int. Ed. 2018, 57, 10765.

[14] Kurpil, B.; Savateev, A.; Papaefthimiou, V.; Zafeiratos, S.; Heil, T.; Özenler, S.; Dontsova, D.; Antonietti, M. Appl. Catal., B 2017, 217, 622.

[15] Poizot, P.; Gaubicher, J.; Renault, S.; Dubois, L.; Liang, Y.; Yao, Y. Chem. Rev. 2020, 120, 6490.

[16] Liang, Y.; Yao, Y. Joule 2018, 2, 1690.

[17] (a) Chen, R.; Luo, R.; Huang, Y.; Wu, F.; Li, L. Adv. Sci. 2016, 3 , 1600051 .

(b) Liu, J.; Zhang, J.-G.; Yang, Z.; Lemmon, J. P.; Imhoff, C.; Graff, G. L.; Li, L.; Hu, J.; Wang, C.; Xiao, J.; Xia, G.; Viswanathan, V. V.; Baskaran, S.; Sprenkle, V.; Li, X.; Shao, Y.; Schwenzer, B. Adv. Funct. Mater. 2013, 23, 929.

[18] Larcher, D.; Tarascon, J. M. Nat. Chem. 2015, 7, 19.

[19] Zhang, C. M.; Huang, Z.; Yang, Y.; Wang, D.; He, D. N. Chin. J. Org. Chem. 2014, 34, 1347 (in Chinese). (张春明，黄昭，杨扬，王丹，何丹农，有机化学, 2014, 34, 1347.)

[20] Wang, R.; Okajima, T.; Kitamura, F.; Matsumoto, N.; Thiemann, T.; Mataka, S.; Ohsaka, T. J. Phys. Chem. B 2003, 107, 9452.

[21] Zhu, L.; Ding, G.; Xie, L.; Cao, X.; Liu, J.; Lei, X.; Ma, J. Chem. Mater. 2019, 31, 8582.

[22] Takayuki, M.; Takayuki, K.; Toyonari, S.; Masaharu, S. Chem. Lett. 2011, 40,750 .

[23] Peng, C.; Ning, G.-H.; Su, J.; Zhong, G.; Tang, W.; Tian, B.; Su, C.;
Yu, D.; Zu, L.; Yang, J.; Ng, M.-F.; Hu, Y.-S.; Yang, Y.; Armand, M.; Loh, K. P. Nat. Energy 2017, 2, 17074.

[24] Wang, J.; Tee, K.; Lee, Y.; Riduan, S. N.; Zhang, Y. J. Mater. Chem. A 2018, 6, 2752.

[25] Xu, F.; Chen, X.; Tang, Z.; Wu, D.; Fu, R.; Jiang, D. Chem. Commun. 2014, 50, 4788 .

[26] Wang, J.; Chen, C. S.; Zhang, Y. ACS Sustainable Chem. Eng. 2018, 6, 1772 .

[27] Zhang, Y.; Riduan, S. N.; Wang, J. Chem.-Eur. J. 2017, 23, 16419.

[28] Li, Q.; Wang, H.; Wang, H.-g.; Si, Z.; Li, C.; Bai, J. ChemSusChem 2020, 13, 2449.

[29] Wang, J.; Lee, Y.; Tee, K.; Riduan, S. N.; Zhang, Y. Chem. Commun. 2018, $54,7681$.

[30] Wang, J.; En, J. C. Z.; Riduan, S. N.; Zhang, Y. Chem.-Eur. J. 2020, $26,2581$.

[31] Xu, S.; Wang, G.; Biswal, B. P.; Addicoat, M.; Paasch, S.; Sheng, W.; Zhuang, X.; Brunner, E.; Heine, T.; Berger, R.; Feng, X. Angew. Chem., Int. Ed. 2019, 58, 849.

[32] Wu, M.; Zhao, Y.; Sun, B.; Sun, Z.; Li, C.; Han, Y.; Xu, L.; Ge, Z.; Ren, Y.; Zhang, M.; Zhang, Q.; Lu, Y.; Wang, W.; Ma, Y.; Chen, Y. Nano Energy 2020, 70, 104498.

[33] (a) Du, Y.; Cui, C. H.; Li Z; Zhang Y.; Jiang H.; Liu, Y. CN $113292725,2021$.

(b) Yang, Y.; Zheng, F.; Xia, G.; Lun, Z.; Chen, Q. J. Mater. Chem. A 2015, 3, 18657.

[34] Cheng, X.-B.; Zhang, R.; Zhao, C.-Z.; Wei, F.; Zhang, J.-G.; Zhang, Q. Adv. Sci. 2016, 3, 1600051.

[35] Mao, M.; Gao, T.; Hou, S.; Wang, F.; Chen, J.; Wei, Z.; Fan, X.; Ji, X.; Ma, J.; Wang, C. Nano Lett. 2019, 19, 6665.

[36] Poizot, P.; Gaubicher, J.; Renault, S.; Dubois, L.; Liang, Y.; Yao, Y. Chem. Rev. 2020, 14, 6490

[37] Mao, M.; Luo, C.; Pollard, T. P.; Hou, S.; Gao, T.; Fan, X.; Cui, C.; Yue, J.; Tong, Y.; Yang, G.; Deng, T.; Zhang, M.; Ma, J.; Suo, L.; Borodin, O.; Wang, C. Angew. Chem., Int. Ed. 2019, 58, 17820.

[38] Shi, R.; Liu, L.; Lu, Y.; Wang, C.; Li, Y.; Li, L.; Yan, Z.; Chen, J. Nat. Commun. 2020, $11,178$.

[39] Liang, Y.; Jing, Y.; Gheytani, S.; Lee, K.-Y.; Liu, P.; Facchetti, A.; Yao, Y. Nat. Mater. 2017, 16, 841.

[40] Wu, X.; Hong, J. J.; Shin, W.; Ma, L.; Liu, T.; Bi, X.; Yuan, Y.; Qi, Y.; Surta, T. W.; Huang, W.; Neuefeind, J.; Wu, T.; Greaney, P. A.; Lu, J.; Ji, X. Nat. Energy 2019, 4, 123.

[41] Tie, Z.; Liu, L.; Deng, S.; Zhao, D.; Niu, Z. Angew. Chem., Int. Ed. 2020, 59, 4920.

[42] (a) Ye, H. Y.; Li, W.; Li, W. S. Chin. J. Org. Chem. 2012, 32, 266 (in Chinese)

(叶怀英, 李文, 李维实, 有机化学, 2012, 32, 266.)

(b) Shao, J. Y.; Zhong, Y. W. Chin. J. Org. Chem. 2021, 41, 1447 (in Chinese). (邵将洋, 钟羽武, 有机化学, 2021, 41, 1447.)

[43] Choudhary, S.; Gozalvez, C.; Higelin, A.; Krossing, I.; MelleFranco, M.; Mateo-Alonso, A. Chem.-Eur. J. 2014, 20, 1525.

[44] Zhao, D.; Zhu, Z.; Kuo, M.-Y.; Chueh, C.-C.; Jen, A. K.-Y. Angew. Chem., Int. Ed. 2016, 55, 8999.

[45] Zhu, R.; Li, Q.-S.; Li, Z.-S. ACS Appl. Mater. Inter. 2020, 12, 38222 .

[46] Zhang, L. L.; Zhao, X. S. Chem. Soc. Rev. 2009, 38, 2520.

[47] Kou, Y.; Xu, Y.; Guo, Z.; Jiang, D. Angew. Chem., Int. Ed. 2011, 50, 8753.

[48] Yan, R.; Leus, K.; Hofmann, J. P.; Antonietti, M.; Oschatz, M. Nano Energy 2020, 67, 104240.

[49] Manthiram, A.; Song, B.; Li, W. Energy Stor. Mater. 2017, 6, 125.

[50] Jerng, S. E.; Chang, B.; Shin, H.; Kim, H.; Lee, T.; Char, K.; Choi, J. W. ACS Appl. Mater. Inter. 2020, 12, 10597. 